\title{
COUNTING STATISTICS MODIFIED BY TWO DEAD TIMES IN SERIES
}

\author{
H. D. CHOI \\ Department of Nuclear Engineering, Seoul National University \\ 599 Gwanak-ro, Gwanak-gu, Seoul 151-744, Republic of Korea \\ *Corresponding author. E-mail : choihdg@snu.ac.kr \\ Received October 25, 2010 \\ Accepted for Publication January 12, 2011
}

Counting statistics modified by introducing two dead times in series under a Poisson input distribution are discussed. A previous study examined the two cases of series combinations of nonextended-extended (NE-E) and extended-extended (EE) dead times. The present study investigated the remaining two cases of extended-nonextended (E-NE) and nonextendednonextended (NE-NE) dead times. For the three time origins of the counting processes - ordinary, equilibrium, and shifted processes - a set of formulae was newly developed from a general formulation and presented for the event time interval densities, total densities, and exact mean and variance of the counts in a given counting duration. The asymptotic expressions for the mean and variance of the counts, which are most convenient for applications, were fully listed. The equilibrium mean count rates distorted by the three dead times in series were newly derived from the information obtained in these studies. An application of the derived formulae is briefly discussed.

KEYWORDS : Nuclear Counting; Counting Statistics; Dead Time, Dead Times in Series

\section{INTRODUCTION}

The dead times that occur in counting systems cause a loss of counts. For the original process, described by a simple Poisson distribution, the counting statistics are so simple that the event intervals are of an exponential type and the variance of counts is equal to the mean number of counts, independent of the counting period. The dead times distort the counting statistics, including the event interval distribution, mean and variance of the counts, and other quantities as well. Since the topic of counts loss is mostly important for radiation detection in nuclear science and technology, a lot of effort has been made to understand and correct the effects of dead time (DT). Earlier studies have modeled and classified the DTs into the extended (E, paralyzable or cumulative) types and non-extended (NE, non-paralyzable or non-cumulative) types [1,2]. The E-type DT is initiated by any arriving pulse and is extended for its magnitude $\tau_{\mathrm{E}}$, beginning from the instant of pulse arrival. The NE-type DT is activated by an arriving pulse only when the pulse arrives at the DT circuit in a state free from dead status, but its period is fixed to the magnitude $\tau_{\mathrm{NE}}$, and is not extended by any pulse arriving during the DT period. No pulses arriving during the DT period are allowed to pass the circuit nor can they register a count [3-5]. The description of DTs by either an E-type or an NE-type has been studied extensively to determine the effects of the DT on the counting statistics. After a few pioneering studies spanning more than a half-century, the detailed statistics of counting are known for a single DT of NE- or E-type under a Poisson input distribution [1-2,6-16]. For this progress, the development and use of renewal theory and operational calculus is crucial $[1,2,17]$. In theoretical modeling of DT behavior, an event pulse is regarded as infinitely sharp in the time domain. Real pulses have, however, finite widths and hence they can be overlapped and lead to pulse pileup. In addition to E- and NE-DTs, pulse pile-up is another important source of counting loss and distortion of statistics. However, pulse pile-up has a complicated DT feature depending on the details of the counting system, as well as the pulse heights in pile-up. Pommé et al. demonstrated that the counting statistics of leading edge pile-up are fundamentally different from those of the E- and NE-type DTs. Therefore, it is known as a new type for describing the DT [18]. The effects of pulse pile-up have been studied in the specific experimental arrangement [18], whereas more theoretical work is still needed to fully understand the statistics. For an experimental study on counting statistics distorted by DTs, most of the concern has concentrated on correcting the count loss due to the DTs present in the detection system. Many experimental techniques have been developed for this goal, including the live time method $[19,20]$, pulser technique $[21,22]$, 
loss-free counting [23,24], and the real time method by imposing an artificial but controlled DT $[25,26,28]$ with a DT correction for counting loss based on a transmission formula $[25,27]$. Additional information on the variance of the counts modified by the DTs is required for measurements based on the variance-to-mean ratio [28-31]. This is also important for accessing the uncertainty of DT correction based on the transmission formula $[32,33]$.

Various experimental approaches can be used to characterize the DTs in a counting system. A review of the experimental problems associated with DT measurement is beyond the scope of this study and reference to the relevant reports is recommended [3,34]. The most basic and characteristic information on DTs in a counting system is obtained by measuring the event time interval distribution (TID). Few studies have successfully cleared the DT characteristics from the measured TID in the detection arrangement, particularly when the series DTs were involved and the theoretical TID distorted by the series DTs was unknown $[13,14,18,31,35]$. Characterization of the DT and accessing of its magnitude is facilitated by inserting a DT module, which can provide either an NEtype [25] or E-type DT [26] with a controllable duration, at the later stage of the counting circuit $[25,27,28,31]$. Alternatively, the mean counting rates or variances are measured for various input rates and the distorted behavior of the counting statistics is compared with the DT model prediction. Even if the TID is measured, it normally indicates the magnitude of the dominant DT in the counting arrangement, whereas modeling the counting behavior by either a single E or NE DT is insufficient for a realistic system to explain the counting rate or variance, especially at higher counting rates. DT models based on a series combination of two or more DTs are more suitable because a real counting system is normally composed of a detector and several electronic circuit modules combined in series $[1,14,27,33,36-41]$. An exact analysis of the DT behavior of the counting arrangement requires a general theoretical treatment of many DTs occurring in series. In this context, the counting statistics of two DTs in series on the starting approach were studied long ago. There are four possible cases of two DTs combined in series; NE-NE, E-NE, NE-E and E-E. Earlier theoretical studies focused mainly on calculating the mean count rates or, more precisely, the asymptotic mean count rates [1,6,14,36-39]. These theoretical studies of series of two DTs did not lead to an understanding of the full counting statistics, due mainly to complications involved in the relevant mathematics. To probe the behavior of counting statistics for such a complicated DT system, the Monte Carlo simulation provides a versatile application and can be used as an alternative. If mathematical analysis can be achieved successfully, it will provide a firm basis and exact formulae to understand the distorted statistics of the counting system and to correct the experimental effects caused by the DTs. For a detailed discussion of the counting statistics, the
TID functions, the exact mean and variance of the counts, and the asymptotic mean and variance of counts in a measurement interval, were considered. These five observable statistics need to be classified for the three different choices to start the counting period, "ordinary," "equilibrium (or stationary)," and "shifted (or free counter)" processes [15]. In the complete description of the counting statistics, there are also quantities, such as the first event TID function, $g_{1}(\mathrm{t})$, for the equilibrium counting and the probability, $W_{\mathrm{k}}(\mathrm{t})$, for obtaining the exact $\mathrm{k}$ counts within the counting period $(0, t)$.

In a previous study, the event TID functions, the exact mean and variance of the counts in a measurement interval, and the associated asymptotic expressions were given in a full set for a series of two DTs ended by an E-type DT, which were denoted as NE-E and E-E cases, respectively [40]. Most of the derivations were based on the existing formalism arising from renewal theory and operational calculus with associated tedious algebra [1,2,9-17]. An explicit derivation can be avoided for the first event TID function, $g_{1}(\mathrm{t})$, of the equilibrium process and the probability distribution, $W_{\mathrm{k}}(\mathrm{t})$, of exact $\mathrm{k}$ counts in time $\mathrm{t}$, in order to obtain the exact mean and variance of the counts [40]. Instead, these values could be obtained from an integral formula that leads to a closed form or a numerical one. In this study, the event TID functions, the exact mean and variance of the counts in a measurement interval, and the associated asymptotic expressions were given in a full set for the series of two DTs ended by an NE-type DT, which were denoted by E-NE and NE-NE cases, respectively. The features of the counting statistics were discussed for each case of the E-NE and NE-NE series DTs. Through the present and previous studies [40], all the TID functions and total density functions were known for the series of two DTs. Therefore the advanced task of providing the mean counting rates for the series of three DTs was achieved.

\section{MATHEMATICAL THEORY}

\subsection{The Event TID and Total Density}

Fig. 1 gives a schematic diagram of the series combination of two DTs. At a point in the circuit, the arrival of events or pulses is random due to the random nature of these events at the input of the detection system. In an ordinary process of counting, the time origin is taken as the instant of an event arriving at a position in the circuit under consideration; the event is not taken into a count but is regarded as the zeroth event by convention. Then, the interval between the successive events is a random variable of which the density distribution function is the event TID function $f(\mathrm{t})$. This is the most fundamental quantity that needs to be obtained before considering all the other quantities. The interval between the $\mathrm{k}$-fold successive events is the multiple interval density of the 
order $\mathrm{k}$, which is denoted by $f_{\mathrm{k}}(\mathrm{t})$. It is simple to see that $f_{1}(\mathrm{t})=f(\mathrm{t})$, and $f_{\mathrm{k}}(\mathrm{t})(\mathrm{k} \geq 2)$ is given by the convolution integral of $f_{\mathrm{k}-1}(\mathrm{t})$ and $f(\mathrm{t})$. An equally important quantity is the total density (or renewal density) function, $D(\mathrm{t})$, which is the rate of arriving events at time $t$. Since the arriving event rate at time $t$ is comprised of all the possible $\mathrm{k}$-th fold successive events, the total density function, $D(\mathrm{t})$, is given by the sum,

$$
D(t)=\sum_{k=1}^{\infty} f_{k}(t)
$$

It is well-known that instead of performing the k-fold convolution integral to obtain $f_{\mathrm{k}}(\mathrm{t})$, the operational calculus based on the Laplace transformation and its inverse is taken from the tables to obtain the final result in a straightforward manner [42]. The Laplace-transformed quantities are denoted by both a right superscript * and the variable s, such as $f_{\mathrm{k}}{ }^{*}(\mathrm{~s})$, and $D^{*}(\mathrm{~s})$. Using the Laplace transform, $f^{*}(\mathrm{~s})$, the total density in the s-domain, $D^{*}(\mathrm{~s})$, can be given as follows:

$$
D^{*}(s)=\frac{f^{*}(s)}{1-f^{*}(s)} .
$$

The inverse of the $D^{*}(\mathrm{~s})$ gives the total density, $D(\mathrm{t})$, often after some lengthy algebra. Eq. (2) holds true between any TID function $f^{*}(\mathrm{~s})$ and the corresponding total density function $D^{*}$ (s) [17]. The location of the circuit at which the densities are relevant is indicated by the left subscript. The originating event TID was assumed to be ${ }_{0} f(\mathrm{t})=$ $\mathrm{U}(\mathrm{t}) \rho \exp (-\rho \mathrm{t})$, a Poisson distribution [5] with a constant event rate, ${ }_{0} D(\mathrm{t})=\rho \mathrm{U}(\mathrm{t})$, where $\mathrm{U}(\mathrm{x})$ is the Heaviside unit step function [42]. The DT modifies the TID and counting statistics of the incoming events. The left superscript NE, E or their combination in the modified statistics indicates the type of the first DT or that of the series DTs. When the magnitude of the second DT is less than or equal to that of the first one $\left(\tau_{2} \leq \tau_{1}\right)$, it does not affect the overall counting and statistics (masking effect). Hence, only the case of $\tau_{2}>\tau_{1}$ is considered in the series DT model. The TID and total density function of the pulse events modified by the first DT and their Laplace transforms are well established and are listed in Table 1 [1,2,9,12-14,38]. Fig. 2 shows the shape of the TID and total density functions in Table 1 .

The effect of an NE DT (the second DT in Fig. 1) for a given arbitrary input distribution of pulse intervals is described by the master equation in several equivalent forms as follows $[1,9,10,14,33]$ :

$$
\begin{aligned}
f(t) & =U\left(t-\tau_{2}\right)\left[{ }_{1} f(t)+\int_{0}^{\tau_{2}}{ }_{1} D(\lambda)_{1} f(t-\lambda) d \lambda\right] \\
& =U\left(t-\tau_{2}\right)\left[{ }_{1} D(t)-\int_{\tau_{2}}^{t} D(\lambda)_{1} f(t-\lambda) d \lambda\right] \\
& =U\left(t-\tau_{2}\right)_{1} D(t)-\int_{0}^{t} U\left(\lambda-\tau_{2}\right)_{1} D(\lambda)_{1} f(t-\lambda) d \lambda .
\end{aligned}
$$

The same equation can also be used to verify ${ }^{\mathrm{NE}} f(\mathrm{t})$ for the Poisson input only if the subscript 2's and 1's are changed to 1's and 0's, respectively, and by consulting the corresponding quantities in Table 1.

The TID function for the E-NE DTs has been derived by many authors and is well established [33,36,43]. It is simply the ${ }^{\mathrm{E}_{1}} f\left[\mathrm{t}-\left(\tau_{2}-\tau_{1}\right)\right]$, which is equal to the ${ }^{\mathrm{E}_{1}} f(\mathrm{t})$ translated by a magnitude of $\tau_{2}-\tau_{1}$ [33]. As shown in Table 1 , the TID of the Poisson process modified by a single NE DT has a similar translation feature, such that its TID is given by ${ }_{1}{ }_{1} f(\mathrm{t})=\mathrm{U}\left(\mathrm{t}-\tau_{1}\right) \rho \exp \left[-\rho\left(\mathrm{t}-\tau_{1}\right)\right]={ }_{0} f\left(\mathrm{t}-\tau_{1}\right)$. The common property according to which the modified TID function is given by the translated one of the input TID of each case, of $f\left(\mathrm{t}-\tau_{1}\right)$ and ${ }^{\mathrm{E}}{ }_{1} f\left[\mathrm{t}-\left(\tau_{2}-\tau_{1}\right)\right]$ respectively, is due to the fact that the corresponding total density $D(\mathrm{t})$ of their input is constant in the time region after the DT of the preceding stage. This can be proven using the master equation (3), which is applied for an NE-type DT of magnitude $\tau_{\mathrm{N}}$, and by assuming the input's total density, $D(\mathrm{t})$, of the form $\mu \mathrm{U}(\mathrm{t}-\tau)$, i.e. a constant $\mu$ after time $\tau(\tau$ $\geq 0)$. The input total density indicates in general that its TID function is of the form, $\mathrm{U}(\mathrm{t}-\tau) f(\mathrm{t})$. No incoming density $D(\mathrm{t})$ in time $\mathrm{t}<\tau$ requires the condition $\tau_{\mathrm{N}}>\tau$ for the statistics to be modified (no masking effect). The

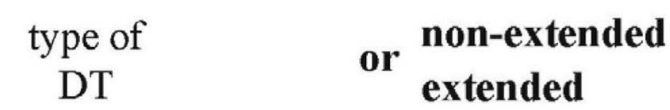

\section{non-extended}

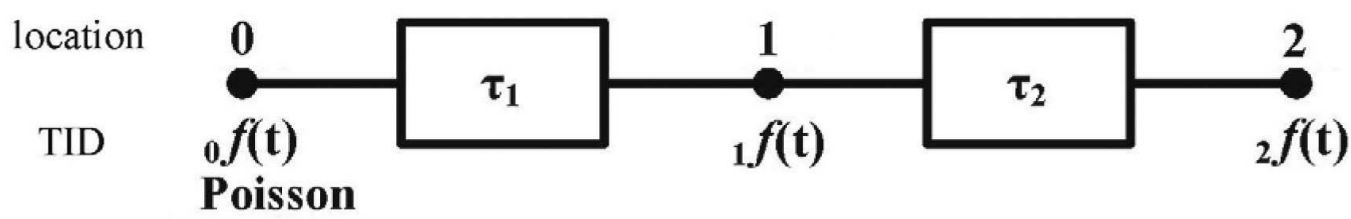

asymptotic mean rate $\rho$ $\mathbf{R}_{\mathbf{1}} \quad \mathbf{R}_{\mathbf{2}}$

Fig. 1. A Schematic Block Diagram of the Two DTs in Series. $\tau_{2}:$ DT of NE-type, $\tau_{1}:$ DT of Either E- or NE-type. TID: Time Interval Density Distribution. 

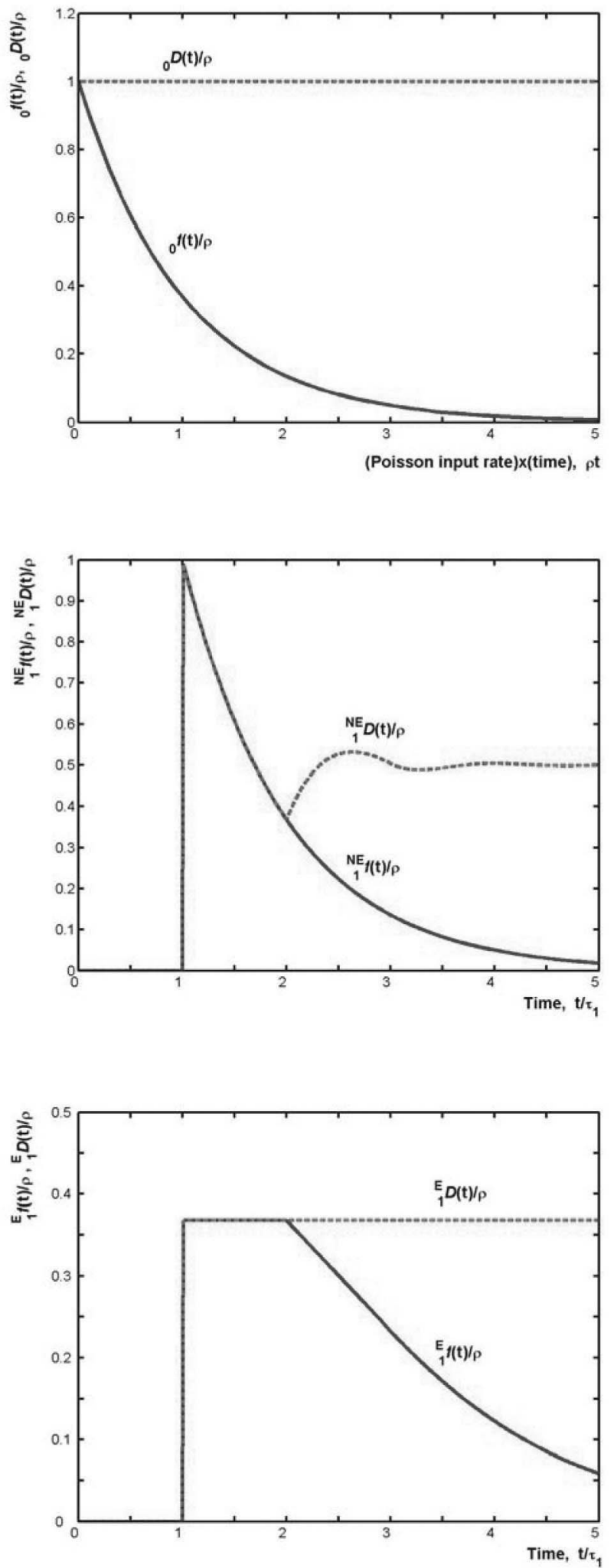

Fig. 2. (Color Online) The Event TIDs, Total Density Functions for the Poisson Process (Top), and the Processes Modified by an NE DT (Middle) or an E DT (Bottom) for the Case of $\rho \tau_{1}=1.0$. subscripts indicating the positions are no longer relevant because Eq. (3) is generally valid for an arbitrary input. Hence, the event TID, ${ }_{m} f(\mathrm{t})$, which is modified from the input TID, $f(\mathrm{t})$, is given by the following:

$$
\begin{aligned}
{ }_{m} f(t) & =U\left(t-\tau_{N}\right) \mu U(t-\tau) \\
& -\mu \int_{0}^{t} U\left(\lambda-\tau_{N}\right) U(\lambda-\tau) f(t-\lambda) d \lambda \\
& =\mu U\left(t-\tau_{N}\right)-\mu \int_{0}^{t} U\left(\lambda-\tau_{N}\right) f(t-\lambda) d \lambda .
\end{aligned}
$$

Using the Laplace transform, $\mathrm{L}\{\mu \mathrm{U}(\mathrm{t}-\mathrm{k}) ; \mathrm{s}\}=\mu \exp (-\mathrm{ks}) / \mathrm{s}$ $(k \geq 0)$ [42], the Laplace transformed functions in Eq. (4) can be obtained as follows:

$$
\begin{aligned}
{ }_{m} f^{*}(s) & =L\left\{\mu U\left(t-\tau_{N}\right) ; s\right\} \\
& -\mu L\left\{\int_{0}^{t} U\left(\lambda-\tau_{N}\right) f(t-\lambda) d \lambda ; s\right\} \\
& =\frac{\mu}{s} e^{-\tau_{N} s}-\frac{\mu}{s} e^{-\tau_{N} s} f^{*}(s)=\frac{\mu}{s} e^{-\tau_{N} s}\left[1-f^{*}(s)\right] \\
& =\frac{\mu e^{-\tau_{N} s}}{s} \frac{f^{*}(s)}{D^{*}(s)}=e^{-\left(\tau_{N}-\tau\right) s} f^{*}(s)
\end{aligned}
$$

where Eq. (2) is used in the second line. Using the Laplace transform, $\mathrm{L}\{\mathrm{U}(\mathrm{t}-\mathrm{k}) f(\mathrm{t}-\mathrm{k}) ; \mathrm{s}\}=\exp (-\mathrm{ks}) f^{*}$ (s) [42], the inverse transform of Eq. (5) gives ${ }_{\mathrm{m}} f(\mathrm{t})=\mathrm{U}\left[\mathrm{t}-\left(\tau_{\mathrm{N}}-\tau\right)\right] f$ $\left[\mathrm{t}-\left(\tau_{\mathrm{N}}-\tau\right)\right]=\mathrm{U}\left(\mathrm{t}-\tau_{\mathrm{N}}\right) f\left[\mathrm{t}-\left(\tau_{\mathrm{N}}-\tau\right)\right]$. The TID of the Poisson process modified by a single NE DT is verified for ${ }^{\mathrm{NE}}{ }_{1} f$ $(\mathrm{t})={ }_{0} f\left(\mathrm{t}-\tau_{1}\right)$ by letting $\tau_{\mathrm{N}}=\tau_{1}$ and $\tau=0$. The case of E-NE is also verified by letting $\tau_{N}=\tau_{2}$ and $\tau=\tau_{1}$.

The TID function and total density for the NE-E DTs were derived in a previous study [40]. The total density, ${ }^{\mathrm{NE}-\mathrm{E}}{ }_{2} D(\mathrm{t})$, is given by the following:

$$
\begin{aligned}
{ }_{2} D(t)= & e^{-\rho\left(\tau_{2}-\tau_{1}\right)} \sum_{j=1}^{J_{t}} U\left(t-\tau_{2}+\tau_{1}-j \tau_{1}\right) e^{-\rho\left[t-\tau_{2}+\tau_{1}-j \tau_{1}\right]} \\
& \rho^{j}\left[t-\tau_{2}+\tau_{1}-j \tau_{1}\right]^{j-1} /(j-1) ! \\
= & e^{-\rho\left(\tau_{2}-\tau_{1}\right) N E} D\left(t-\tau_{2}+\tau_{1}\right)
\end{aligned}
$$

This is simply the input total density ${ }^{\mathrm{NE}}{ }_{1} D(\mathrm{t})$ translated by the magnitude of $\tau_{2}-\tau_{1}$ and modified by a factor of $\exp (-$ $\left.\rho \tau_{2}+\rho \tau_{1}\right)$. Similarly the total density ${ }_{1}{ }_{1} D(\mathrm{t})=\mathrm{U}\left(\mathrm{t}-\tau_{1}\right) \rho \exp (-$ $\left.\rho \tau_{1}\right)$ is the Poisson total density $\rho U(t)$ translated by $\tau_{1}$ and modified by a factor of $\exp \left(-\rho \tau_{1}\right)$. This is a general feature arising for an E-type DT, whenever the input TID function, $f(\mathrm{t})$, is of the form $\mathrm{U}(\mathrm{t}-\mathrm{k}) \mathrm{ae}^{-\mathrm{bt}}(\mathrm{a}>0, \mathrm{~b}, \mathrm{k} \geq 0)$. Again, no incoming density $f(\mathrm{t})$ in time $\mathrm{t}<\mathrm{k}$ requires the condition $\tau_{\mathrm{E}}>\mathrm{k}$ for the statistics to be modified. Using the master equation for E-type DT with magnitude $\tau_{\mathrm{E}}$, the total density, ${ }_{\mathrm{m}} D(\mathrm{t})$, modified from the input total density, $D(\mathrm{t})$, can be given by the following $[1,40]$ :

$$
{ }_{m} D(t)=U\left(t-\tau_{E}\right) f(t)+\int_{0}^{t} D(t-\lambda) f(t) d \lambda .
$$


The Laplace transformed functions in Eq. (7) are given by

$$
\begin{aligned}
{ }_{m} D^{*}(s) & =\frac{L\left\{U\left(t-\tau_{E}\right) f(t) ; s\right\}}{1-f^{*}(s)} \\
& =\frac{D^{*}(s)}{f^{*}(s)} L\left\{U\left(t-\tau_{E}\right) f(t) ; s\right\}=D^{*}(s) e^{-(s+b)\left(\tau_{E}-k\right)}
\end{aligned}
$$

where the Laplace transforms, $f^{*}(\mathrm{~s})=\mathrm{L}\left\{\mathrm{U}(\mathrm{t}-\mathrm{k}) \mathrm{ae}^{-\mathrm{bt}}\right.$; $\mathrm{s}\}=\mathrm{ae}^{-(\mathrm{s}+\mathrm{b}) \mathrm{k}} /(\mathrm{s}+\mathrm{b})$, and $\mathrm{L}\left\{\mathrm{U}\left(\mathrm{t}-\tau_{\mathrm{E}}\right) f(\mathrm{t}) ; \mathrm{s}\right\}=\mathrm{ae}^{-(\mathrm{s}+\mathrm{b}) \tau_{\mathrm{E}}} /(\mathrm{s}+\mathrm{b})$, were obtained by consulting the table [42]. Hence, the inverse transform gives

$$
{ }_{m} D(t)=U\left[t-\left(\tau_{E}-k\right)\right] D\left(t-\tau_{E}+k\right) e^{-b\left(\tau_{E}-k\right)} .
$$

The total density of the Poisson process modified by the series of NE-E DTs is verified by letting $\tau_{\mathrm{E}}=\tau_{2}, \mathrm{k}=\tau_{1}$, $a=\rho \exp \left(\rho \tau_{1}\right)$, and $b=\rho$ in Eq. (9), which proves the relation in Eq. (6). Similarly, the total density of the Poisson process modified by an E DT of magnitude $\tau_{1}$, is also verified by letting $\tau_{\mathrm{E}}=\tau_{1}, \mathrm{k}=0, \mathrm{a}=\mathrm{b}=\rho$ in Eq. (9).

\subsection{The Counting Statistics}

Since the time origin of the start, in an ordinary process of counting, is taken with a pulse arriving at the output, the TID function $f_{1}(\mathrm{t})$ for the first event is obviously the TID function $f(\mathrm{t})$. In an equilibrium process, the time origin of counting is taken at random. Hence, the TID function for the first event, $g_{1}(\mathrm{t})$, is obtained from its relation to the TID function $f(\mathrm{t})[14,17]$. A previous study [40] showed that obtaining an explicit form of the $g_{1}(\mathrm{t})$ function can be avoided because all the moments of the counting that are relevant to the equilibrium process can be obtained from the TID function, $f(\mathrm{t})$, and the total density function, $D(\mathrm{t})$, using Laplace transformation methods. In addition, the probability, $W_{\mathrm{k}}(\mathrm{t})$, of obtaining exactly $\mathrm{k}$ counts within time $t$ need not be listed. These quantities, in the cases of two DTs in series, take not only complicated forms but require complicated mathematics to obtain the mean values and variances. Alternatively and exclusively, the Laplace transformed quantities and their inversions are used with algebra to rearrange the terms. The expected number of counts $E(k, t)$ in the time interval $(0, t)$ can be given by the following:

$$
E(k, t)=L^{-1}\left\{\frac{{ }_{2} D^{*}(s)}{s}\right\}=\int_{0}^{t}{ }_{2} D(\lambda) d \lambda
$$

for an ordinary process. For an equilibrium process, an exact, general and simple relation is already known as $[14,17]$ :

$$
E(k, t)=\mu t, \quad \mu^{-1}=\int_{0}^{\infty} 2 f(t) t d t=-\left.\frac{d_{2} f^{*}(s)}{d s}\right|_{s=0}
$$

where the second equalities are based on the Elmore theorem [44]. An alternative method exists to obtain the equilibrium mean count rate $\mu$ without addressing it to the TID modified by the relevant DT. For a counting process modified by an NE-type DT of magnitude $\tau_{2}$, the mean count rate $\mu_{2}$ can be obtained using a simple equation in terms of the quantities at position- 1 , such as $[1,45]$

\begin{tabular}{|c|c|c|}
\hline Position & Event TID and Total density functions & Laplace transforms \\
\hline \multirow{2}{*}{0} & ${ }_{0} f(t)=U(t) \rho e^{-\rho t}$ & ${ }_{0} f^{*}(s)=\frac{\rho}{s+\rho}$ \\
\hline & ${ }_{0} D(t)=U(t) \rho$ & ${ }_{0} D^{*}(s)=\frac{\rho}{s}$ \\
\hline \multirow{2}{*}{1} & ${ }_{1}^{N E} f(t)=U\left(t-\tau_{1}\right) \rho e^{-\rho\left(t-\tau_{1}\right)}$ & ${ }_{1}^{N E} f^{*}(s)=\frac{\rho e^{-\tau_{1} S}}{s+\rho}$ \\
\hline & ${ }_{1}^{N E} D(t)=\rho \sum_{j=1}^{J_{t}} U\left(t-j \tau_{1}\right) e^{-\rho\left(t-j \tau_{1}\right)} \rho^{j-1}\left(t-j \tau_{1}\right)^{j-1} /(j-1) !$ & ${ }_{1}^{N E} D^{*}(s)=\frac{\rho e^{-\tau}{ }^{s}}{s+\rho-\rho e^{-\tau_{1} s}}$ \\
\hline \multirow[t]{2}{*}{1} & ${ }_{1}^{E} f(t)=\rho \sum_{j=1}^{J_{t}} U\left(t-j \tau_{1}\right) e^{-j \rho \tau_{1}}(-\rho)^{j-1}\left(t-j \tau_{1}\right)^{j-1} /(j-1) !$ & ${ }_{1}^{E} f^{*}(s)=\frac{\rho e^{-\tau_{1}(S+\rho)}}{s+\rho e^{-\tau_{1}(S+\rho)}}$ \\
\hline & ${ }_{1}^{E} D(t)=U\left(t-\tau_{1}\right) \rho e^{-\rho \tau_{1}}$ & ${ }_{1}^{E} D^{*}(s)=\frac{\rho}{s} e^{-\tau_{1}(s+\rho)}$ \\
\hline
\end{tabular}

$$
\mu_{2}=\mu_{1}\left\{1+\int_{0}^{\tau_{2}} D(t) d t\right\}^{-1}
$$

Table 1. The Event TIDs, Total Density Functions and Their Laplace Transforms in the s-domain for the Poisson Process, and the Processes Modified by an NE DT or an E DT. (See Fig. 1 for Diagram.)

Note) $\mathrm{J}_{\mathrm{t}}$ is the Largest Integer Below $\mathrm{t} / \tau_{1}$, and is abbreviated as $\left[\left[\mathrm{t} / \tau_{1}\right]\right]$. 
where the $\mu_{1}$ is the mean count rate and the ${ }_{1} D(\mathrm{t})$ is the total density at position-1. When the counting process is modified by an E-type DT with magnitude $\tau_{2}$, the mean count rate $\mu_{2}$ can be given by the following equation $[1,14,36]$ :

$$
\mu_{2}=\mu_{1}\left[1-\int_{0}^{\tau_{2}} f(t) d t\right]
$$

where the ${ }_{1} f(\mathrm{t})$ is the TID function at position-1. The integration in Eq. (13) corresponds to the probability of the incoming counts lost by the E-type DT [14,36]. Eqs. (12) and (13) are also useful for cross checking the results obtained by Eq. (11).

Work on the shifted (free counter) process is to be found in the literature $[2,9,11]$. An explicit definition of the free counter process is that the time origin of counting is the immediate moment after the dead period [15]. For an NE-type DT, the dead period is fixed in magnitude and hence the time origin is conceptually simple to consider. For an E-type DT, the dead period can be extended by any incoming pulse and the immediate moment after the dead period is a random variable. Two studies have shown that the TID function of the first event, $h_{1}(\mathrm{t})$, in the shifted process is equal to the original Poisson distribution, which is given by $h_{1}(\mathrm{t})=\left.{ }_{1} f(\mathrm{t})\right|_{\tau=0}={ }_{0} f(\mathrm{t})$ for a single DT of either type, NE or E $[46,47]$. When there are two DTs in the series DTs, it has also been shown that the TID function for the first event, $h_{1}(\mathrm{t})$, is equal to the original Poisson distribution, which is given by $h_{1}(\mathrm{t})=\left.{ }_{2} f(\mathrm{t})\right|_{\tau_{1}=\tau_{2}=0}={ }_{0} f(\mathrm{t})$ [40]. Hence, the expected number of counts $\mathrm{E}(\mathrm{k}, \mathrm{t})$ can be obtained by the following [40]:

$$
\begin{aligned}
E(k, t) & =L^{-1}\left\{\frac{{ }_{0} f^{*}(s)}{s}+\frac{{ }_{0} f^{*}(s)_{2} D^{*}(s)}{s}\right\} \\
& =\int_{0}^{t}{ }_{0} f(\lambda) d \lambda+\int_{0}^{t} \int_{0}^{\sigma}{ }_{2} D(\lambda)_{0} f(\sigma-\lambda) d \lambda d \sigma
\end{aligned}
$$

for the shifted process.

By avoiding the complicated algebra involved when using the quantities $g_{1}(\mathrm{t})$ and $W_{\mathrm{k}}(\mathrm{t})$, the variance $\mathrm{V}(\mathrm{k}, \mathrm{t})$ of the number of counts $\mathrm{k}$ within the counting interval $(0, \mathrm{t})$ can be expressed for all three processes in integral form $[12,17,40]$ :

$$
\begin{aligned}
V(k, t) & \equiv \sigma_{k}^{2}(t) \\
& =2 \int_{0}^{t}{ }_{2} D(\lambda) E(k, t-\lambda) d \lambda+E(k, t)-[E(k, t)]^{2}
\end{aligned}
$$

where $E(k, t)$ is the expected number (or mean number) of counts in the corresponding process of the time origin. For the equilibrium process, Eq. (15) can be reduced further to:

$$
V(k, t) \equiv \sigma_{k}^{2}(t)=2 \mu \int_{0}^{t} \int_{0}^{\sigma}{ }_{2} D(\lambda) d \lambda d \sigma+\mu t-[\mu t]^{2}
$$

where $\mu$ is the equilibrium mean count rate given in Eq. (11) $[12,17,40]$. The integrations of the exact variances for the ordinary and shifted processes, which are given by Eq. (15), become too complicated in analytic terms and are left out for numerical integration, which is simpler and can be performed as accurately as required. Asymptotic expressions for the mean counts and variance are valid in the limit of $t / \tau \rightarrow \infty$, whereas they are approximately valid for counting times $\geq \sim 10 \tau_{2}$, which is the range of value typically taken in most counting measurements. Therefore, the asymptotic forms are mostly convenient and useful. They are derived using the known relations in terms of the $\mathrm{r}$-th moments, $\mathrm{m}_{\mathrm{r}}(\mathrm{t})$, of the time interval and by the Elmore theorem $[44,47]$.

Since the mathematical proofs of the derived formulae are lengthy and less interesting for applications, they were omitted and only the results are listed in the Appendices. The statistics for the asymptotic mean and variance of the counts in the counting time $(0, t)$ are listed in the Appendices for each of the three counting origins (ordinary, equilibrium and shifted processes). Most of the abbreviations in the formulae are for convenience the same as those used in previous studies $[14,15,40]$, and are listed in the Appendix.

\section{FEATURES OF STATISTICS}

\subsection{E-NE Series Dead Time}

Figure 3 shows the TID calculated using Eq. (A.4) and the data points produced by the Monte Carlo simulation. It would only be redundant to show that the theoretical TIDs are consistent with the Monte Carlo simulation results. The case $\tau_{1} / \tau_{2}=1$ can be taken as a limiting case of Eq. (A.4), and the corresponding TID is the same as the TID ${ }_{1} f(\mathrm{t})$ given in Table 1 . The TID function for E-NE DTs has the feature that its non-zero magnitude begins from $t$ $=\tau_{2}$; it also has a flat interval in $\tau_{2} \leq \mathrm{t} \leq \tau_{2}+\tau_{1}$. As the $\tau_{1} / \tau_{2}$ ratio becomes zero, the effect of the first DT decreases, and the TID reduces to the shape of ${ }^{\mathrm{NE}} f(\mathrm{t})$ with a DT magnitude of $\tau_{2}$. Figure 4 shows the distortion of the asymptotic mean count rates, $\mathrm{R}_{1}$ and $\mathrm{R}_{2}$, which were observed at the two locations indicated in Fig. 1. Owing to the second DT, the count rate $\mathrm{R}_{2}$ is degraded appreciably around the Poisson rates $\rho \sim 1 / \tau_{1}$, whereas the magnitude of degradation is small when the magnitudes of both DTs are closer to each other.

The equilibrium mean count rate, Eq. (A.12), was derived long ago for E-NE DTs [14,36]. In this study, the full statistics for the three counting processes are obtained, including the exact and asymptotic variances of counting. In Fig. 5, the variance-to-mean ratio, $\sigma_{\mathrm{k}}{ }^{2}(\mathrm{t}) / \mu_{\mathrm{en}} \mathrm{t}$, given in Eqs. (A.12)-(A.13), reveals the distortion in the increasing counting time $t$ for an equilibrium process. The dashed lines in the figure are the asymptotic ratios calculated by Eqs. (A.12) and (A.14). It is well known that the varianceto-mean ratio of the Poisson input distribution is 1.0 independent of the rate, $\rho$, and the counting time, $t$. The 
modified variance-to-mean ratio by the E-NE series DT model is always less than one, and the ratio generally decreases with increasing counting time. For a counting time $\mathrm{t} \geq \sim 10 \tau_{2}$, the difference between the exact variance and the asymptotic one becomes smaller. The computation of the exact variance given by Eq. (A.13) became timeconsuming and showed numerical instability for a larger counting time t. The asymptotic variance, which is given in Eq. (A.14), is more useful for comparing with the experimental data for which the counting time $t$ is typically

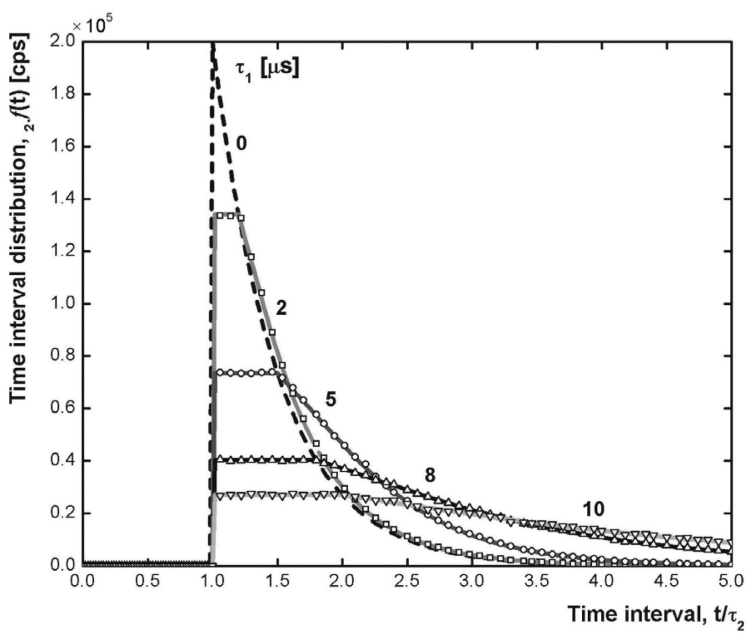

Fig. 3. (Color Online) TIDs for the E-NE Series DTs. The Poisson Input Rate was $\rho=2 \times 10^{5} \mathrm{cps}$, and the Second DT was $\tau_{2}=10 \mu \mathrm{s}$. The Data Points were Produced by the Monte Carlo Simulation.

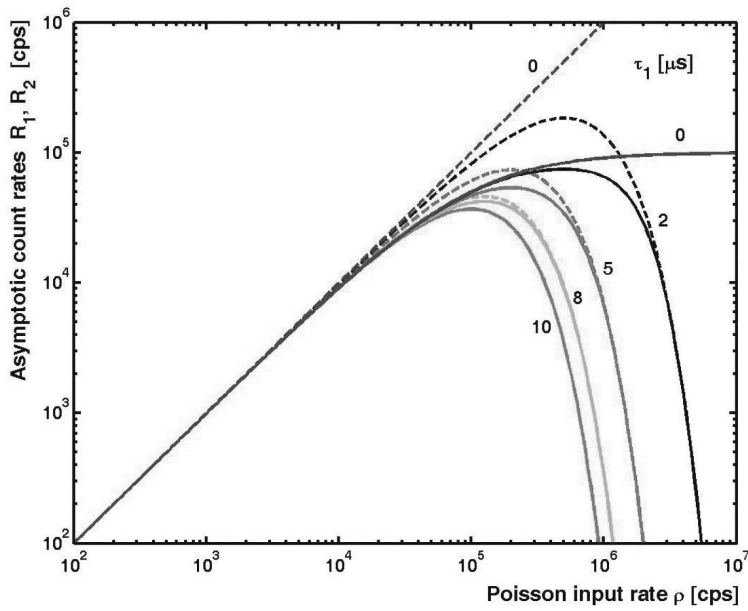

Fig. 4. (Color Online) Mean Count Rate $\mathrm{R}_{1}$ (Dashed Line) after the First DT of the E-type and the Rate $\mathrm{R}_{2}$ (Continuous Line) after the Second DT of the NE-type in a Series of E-NE DTs. The Poisson Input Rate $\rho$ was Varied, while the Second DT was $\tau_{2}=10 \mu \mathrm{s}$. The Cases of $\tau_{1}=0 \mu \mathrm{s}$ and $10 \mu \mathrm{s}$ are Equivalent to Those of a Single DT of the NE- and E-type, Respectively. much larger than $10 \tau_{2}$. The magnitude of the asymptotic variance-to-mean ratio depends on the Poisson input rate, in accordance with the pattern shown in Fig. 6.

A recent experimental application of the variance-tomean ratio can be found in the literature [30,31], in works that examined the subcriticality of a reactor core. Here the variance-to-mean ratios are typically measured by single channel neutron counting consisting of a neutron detector, pre-amplifier, amplifier, discriminator and counter. Through an argument known as the "Feynman $\alpha$

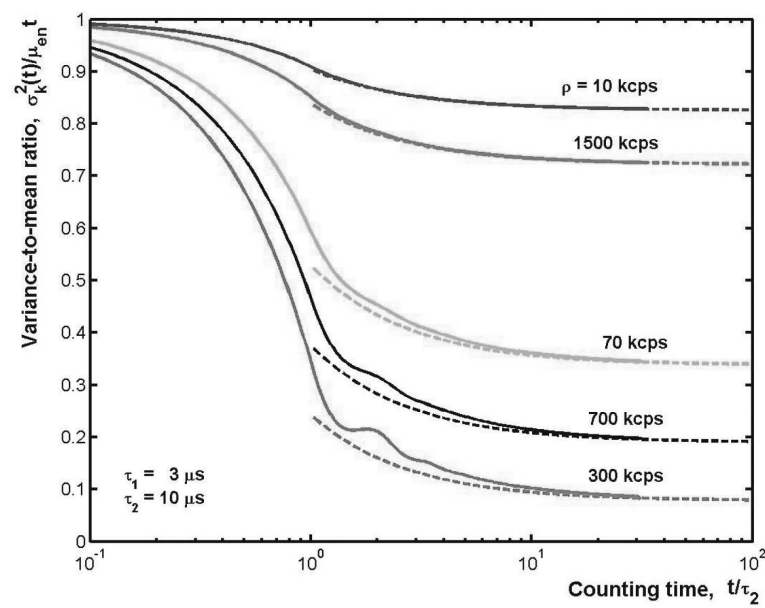

Fig. 5. (Color Online) Exact Variance-to-mean Ratio $\sigma_{\mathrm{k}}^{2}(\mathrm{t}) / \mu_{\mathrm{en}} \mathrm{t}$ for the E-NE Series DTs vs. the Counting Time $t$ Under Equilibrium Counting and for Several Poisson Input Rates $\rho$ (Continuous Line). The first DT, $\tau_{1}=3 \mu \mathrm{s}$, and the Second DT, $\tau_{2}=10$ us, were Fixed. The Asymptotic Variance-to-mean Ratios, which were Based on Eq. (A.14), are Shown in the Dashed Lines.

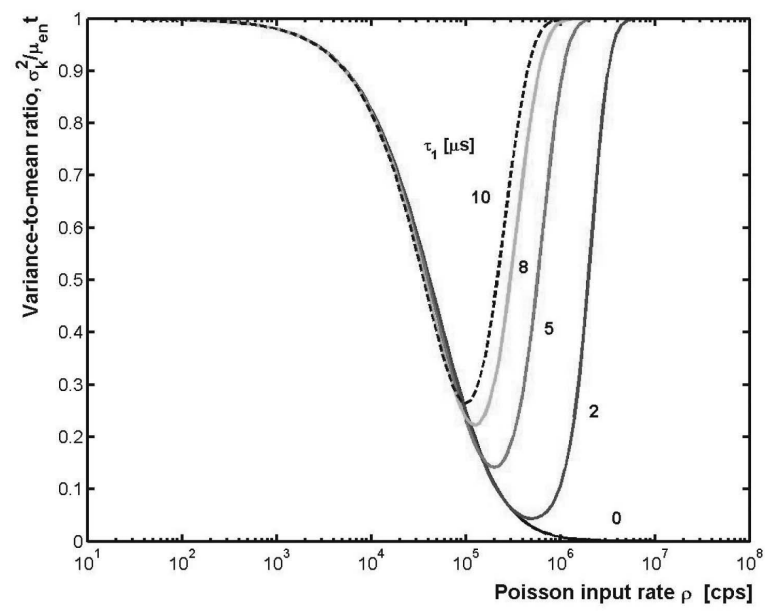

Fig. 6. (Color Online) The Variance-to-mean Ratio $\sigma_{\mathrm{k}}{ }^{2} / \mu_{\mathrm{en}} \mathrm{t}$ for E-NE Series DT Under an Asymptotic Equilibrium Counting. The Poisson Input Rate $\rho$ was Varied, whereas the Second DT, $\tau_{2}=10 \mu \mathrm{s}$, and Counting Time, $\mathrm{t}=1 \mathrm{~s}$, were Fixed for an Asymptotic Limit. 
method", the variance-to-mean ratio of neutron counts in a reactor core is correlated with the prompt neutron decay constant $(\alpha)$ and shutdown reactivity margin [29]. In the experimentally measured variance-to-mean ratios, the deviation from Poisson statistics is affected by the dead time behavior of the detection system, for which the true mean count rate and variance-to-mean ratio are normally estimated by modeling the DT behavior of the detection system. In references $[31,48]$, the measured TID of a single channel neutron counting system had the shape of the typical E-NE DT model, whereas another study in ref. [30], which used a similar counting system, assumed that the DT behavior could be modeled for the NE-E DTs. Both studies need to describe the observed variance-to-mean ratio as the input neutron rate increases. Therefore, the new formulae derived in the present and the previous study [40] are immediately applicable to the experimental data based on the Feynman $\alpha$ method.

\subsection{NE-NE Series Dead Times}

Determination of the mean count rate for the NE-NE series DTs was made long ago by Ruark and Brammer, even though they actually derived that rate for the NE-E series DT model [6]. A correct formula of the equilibrium mean count rate for the NE-NE series DTs was later derived by Jost [1]. After considering a printing error, the equilibrium mean count rate obtained by Jost was confirmed to be equal to the $\mu_{\mathrm{NN}}$ in Eq. (A.3). Müller also considered the problem of the NE-NE series DTs, giving an expression for the TID and equilibrium mean count rate $[14,38]$. Müller's derivation led to a complicated expression for the mean count rate, due to the different approach used to obtain the result. After some work, Müller's formula, Eq. (52) in ref. [14], was found to be equivalent to Jost's [1] and Eq. (A.3) in this study. The TID function ${ }_{2} f(\mathrm{t})$, obtained in this study, is simple, and is given in Eq. (B.1). It was first derived by De Lotto et al. [36] and later by Müller in an equivalent but different form [38]. An expression for ${ }_{2} f(\mathrm{t})$ was previously known, whereas the total density ${ }_{2} D(\mathrm{t})$ given in Eq. (B.2) was newly obtained in this study. Fig. 7 compares the TIDs, $2 f(\mathrm{t})$, with the Monte Carlo simulations. The comparison confirms the validity of the TIDs, ${ }_{2} f(\mathrm{t})$, listed in this study. The shapes of the TIDs, ${ }_{2} f(\mathrm{t})$, shown in Fig. 7, indicate several notable features. The TIDs for $\tau_{1}=0$ and $\tau_{1}=\tau_{2}$ are equal to that of a single DT of the NE-type. All the TIDs show the decay of the characteristic form, $\exp (-\rho t)$, for time $t>\tau_{2}+\tau_{1}$. For high J, e.g. $\tau_{1}=2 \mu \mathrm{s}(\mathrm{J}=5)$, the time region of $\tau_{2}<\mathrm{t}<\tau_{2}+\tau_{1}$, has an almost flat value for ${ }_{2} f(\mathrm{t})$. Here $\mathbf{J}$ is the largest integer below the ratio $\tau_{2} / \tau_{1}$, denoted by $\left[\left[\tau_{2} / \tau_{1}\right]\right]$. The cases of $\tau_{1}=4$ and $5 \mu$ s show a curved region in $\tau_{2}<\mathrm{t}<\tau_{2}+\tau_{1}$. For $\mathrm{J}=1\left(\tau_{1}=6,8 \mu \mathrm{s}\right)$, there is an additional exponential form in the region $\tau_{2}<\mathrm{t}<2 \tau_{1}$. In contrast to the TID, ${ }_{2} f(\mathrm{t})$, the total density function, ${ }_{2} D(\mathrm{t})$, which is given in Eq. (B.2), has a complicated form due to $\mathrm{B}_{\mathrm{rq}}$ 's terms, defined by Eq. (B.5). Fortunately, the definition of $\mathrm{B}_{\mathrm{rq}}$ 's is similar to that of $\mathrm{A}_{\mathrm{rq}}$ 's, which were discussed in a previous paper regarding the E-E series DTs [40]. In the simplest case of $\mathrm{J}=1\left(\tau_{1}<\tau_{2} \leq 2 \tau_{1}\right)$, only one term $\mathrm{q}=\mathrm{r}$ for a given $\mathrm{r}$ is sufficient, such as $\mathrm{B}_{\mathrm{rq}}=\mathrm{B}_{\mathrm{rr}}=\bar{Q}_{1}^{\mathrm{r}}=\exp \left[-\mathrm{r} \rho\left(\tau_{2}-\tau_{1}\right)\right]$, to account for the sum over q. For $J=2\left(2 \tau_{1}<\tau_{2} \leq 3 \tau_{1}\right)$, there are $(\mathrm{r}+1)$ terms for the index $\mathrm{q}$ running from $\mathrm{r}$ to $2 \mathrm{r}$, but they are still given simply as $\mathrm{B}_{\mathrm{rq}}=\bar{Q}_{1}^{2 \mathrm{r}-\mathrm{q}} \bar{Q}_{2}^{\mathrm{q}-\mathrm{r}} \mathrm{r}$ !/(q-r)!(2r-q)!, which can be calculated easily. For a larger $\mathbf{J}$ or $r$, they can be calculated using the recurrence relation in Eq. (B.6). The expected number of counts, $\mathrm{E}(\mathrm{k}, \mathrm{t})$, in a time duration $(0, t)$ and its associated variance, $\mathrm{V}(\mathrm{k}, \mathrm{t})$, in exact or asymptotic form, were newly derived in this study. These were calculated using short computer code with a routine for calculating $\mathrm{B}_{\mathrm{rq}}$.

Figure 8 shows the asymptotic mean count rates, $R_{1}$ and $\mathrm{R}_{2}$, which are observed at the two locations indicated in Fig. 1. The count rate $R_{1}$ approaches the constant count rate $1 / \tau_{1}$ as the input rate $\rho$ is increased. The count rate $R_{2}$ was degraded further due to the second DT but it was not monotonic with increasing input rate $\rho$. The mean counting rate of the NE-NE series DTs for the equilibrium process is $\mu_{\mathrm{NN}}=\rho \lambda_{1} \lambda_{\mathrm{NN}}$, as shown in Eq. (A.3). Here $\rho \lambda_{1}=\rho /\left(1+\rho \tau_{1}\right)$ is the mean counting rate $\left(R_{1}\right)$ for the Poisson input modified by a single DT, $\tau_{1}$, of the NE-type. Hence, the factor $\lambda_{\mathrm{NN}}$ is the transmission factor $\mathrm{T}_{2}$ of the second NE DT for a given input rate, $\rho \lambda_{1}$. The transmission factor $T_{2}$ is given by the following:

$$
\begin{aligned}
T_{2} \equiv \lambda_{N N} & =\left[1+J-\sum_{n=1}^{J} \bar{Q}_{n}\right]^{-1} \\
& =\left\{1+J-\sum_{n=1}^{J} \sum_{j=n}^{J} \frac{\left[\rho\left(\tau_{2}-j \tau_{1}\right)\right]^{j-n}}{(j-n) !} e^{-\rho\left(\tau_{2}-j \tau_{1}\right)}\right\}^{-1} .
\end{aligned}
$$

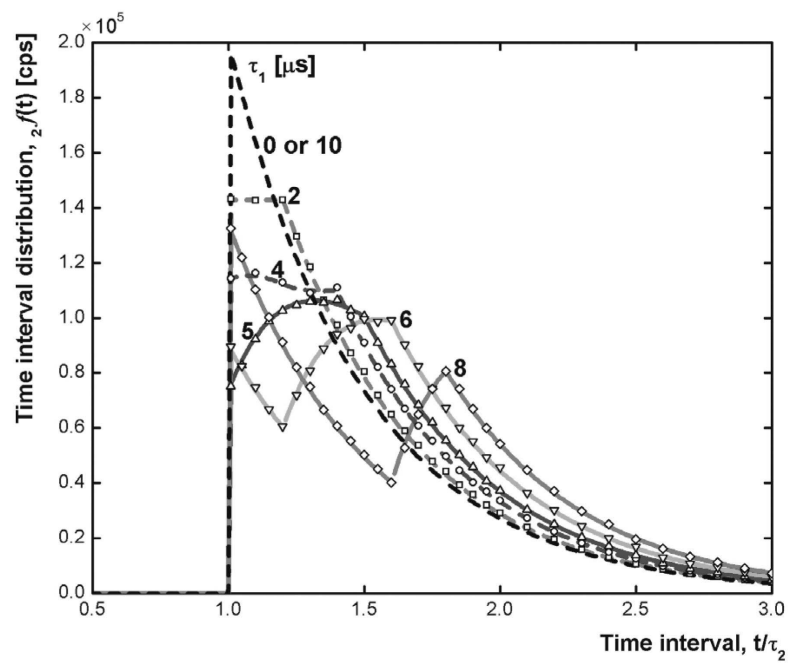

Fig. 7. (Color Online) TIDs Calculated for a Series of NE-NE

DTs. The Input Poisson Rate was $\rho=2 \times 10^{5} \mathrm{cps}$, and the second DT was $\tau_{2}=10 \mu$ s. The Data Points were Produced by the Monte Carlo Simulation. 
In the limit of large $\rho \tau_{2}$, the transmission factor, $T_{2}$, is limited monotonically to $(1+\mathrm{J})^{-1}$, which can be seen in Eq. (17), because all the $\bar{Q}_{n}$ values are diminished. Fig. 9 presents the transmission factor $T_{2}$ at various $\tau_{1} / \tau_{2}$ ratios as a function of the dimensionless rate $\rho \tau_{2}$. For any $\tau_{1}-\tau_{2}$ combination giving the same $\mathrm{J}$, the transmission factor $\mathrm{T}_{2}$ is limited to the same value $(1+\mathrm{J})^{-1}$. Fig. 10 shows the variance-to-mean ratio, $\sigma_{\mathrm{k}}{ }^{2}(\mathrm{t}) / \mu_{\mathrm{NN}} \mathrm{t}$ for an equilibrium process, which is given in Eqs. (B.14)-(B.15). The varianceto-mean ratio was found to deviate from that of the Poisson statistics and to decrease in general with increasing counting time t. In the cases with a large $\rho \tau_{2}$, e.g. lines for $\rho=1$

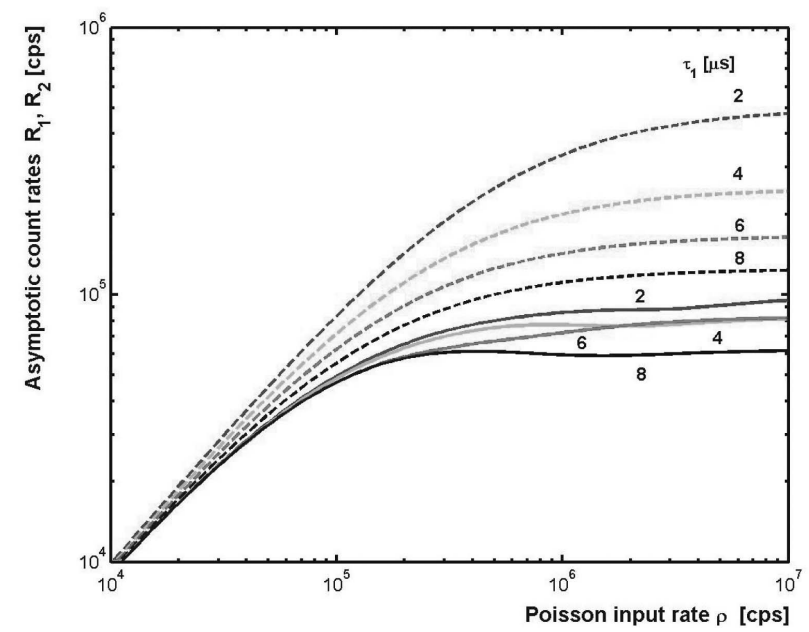

Fig. 8. (Color Online) Asymptotic Mean Count Rate $\mathrm{R}_{1}$ (Dashed Line) after the First DT and the Rate $\mathrm{R}_{2}$ (Continuous Line) after the Second DT in a Series of NE-NE DTs. The Poisson Input Rate $\rho$ was varied, whereas the second DT was taken as $\tau_{2}=10 \mu \mathrm{s}$.

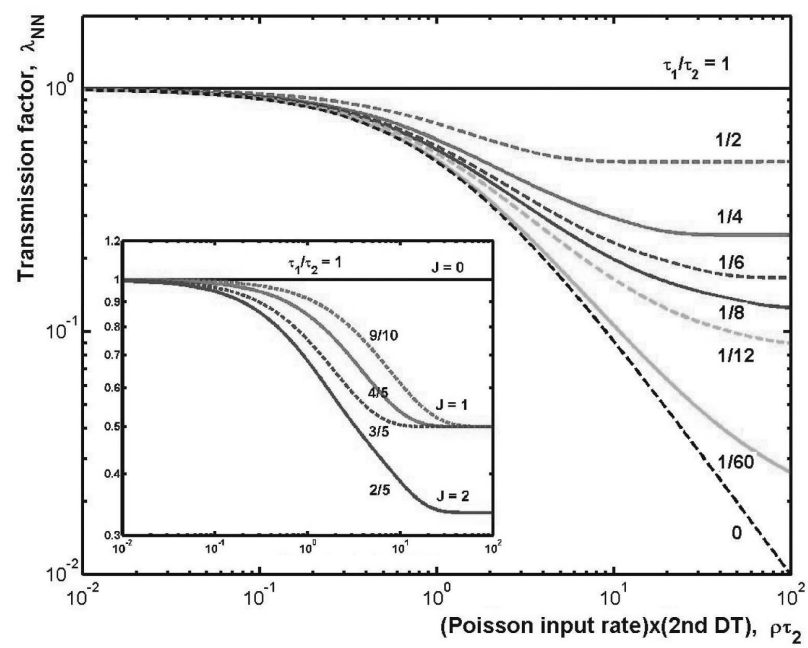

Fig. 9. (Color Online) Transmission factor $T_{2}=\lambda_{N N}$ calculated by Eq. (17) as a function of $\rho \tau_{2}$. and 8 Mcps, shown in Fig. 10, an oscillating feature can be observed for $\mathrm{t}>\tau_{2}$, which is a characteristic feature of the variance modified by the NE-type DT [15]. In Fig. 11, the variance-to-mean ratio $\sigma_{\mathrm{k}}^{2} / \mu_{\mathrm{NN}} \mathrm{t}$, which is given in Eq. (B.16) and Eqs. (B.7)-(B.9), is shown for an asymptotic equilibrium process $(t=1 \mathrm{~s})$ and for several $\tau_{1}$ 's. This illustrates the effect of the second DT of NE-type, which causes a variation from the simple monotonic decrease in the case of a single NE DT.

\subsection{Mean Counting Rate Modified by Three Dead Times in Series}

Counting statistics modified by three DTs in series has never been examined before. The required full set of TID functions and total densities for the two DTs in series was not known previously. Moreover, the mathematics involved in some case was too complicated to make it a topic of interest. Although the counting statistics for the two series DTs are known through our work, the challenging topic of counting statistics modified by three DTs in series is difficult to solve because the mathematics is even more complicated. The throughput formula for equilibrium counting can be simply derived using the known TIDs and total densities for the two DTs in series.

Eqs. (12) and (13) are generally valid for any position in the counting circuit. When they are applied to the counting rate at position-1, of which DT modifies the original Poisson process, the integration is simple and the familiar counting rates can be obtained for the distortion by an E- or an NE-type DT $[1,9,14]$. The equilibrium mean counting rates $\mu_{\mathrm{en}}$ and $\mu_{\mathrm{NN}}$, given in Eqs. (A.2)-(A.3),

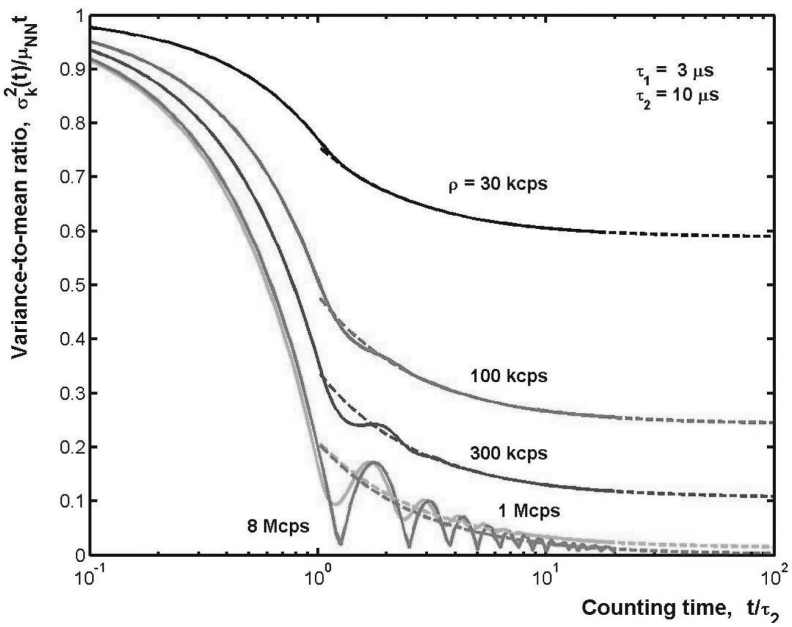

Fig. 10. (Color Online) Exact Variance-to-mean Ratio $\sigma_{\mathrm{k}}{ }^{2}(\mathrm{t}) / \mu_{\mathrm{NN}} \mathrm{t}$ for the NE-NE Series DTs vs. the Counting Time t, Under an Equilibrium Counting and for Several Poisson Input Rates $\rho$. The First DT, $\tau_{1}=3 \mu \mathrm{s}$, and the Second DT, $\tau_{2}=10 \mu \mathrm{s}$, were Fixed. The Asymptotic Variance-to-mean Ratios, which were Based on Eq. (B.16), are Shown in the Dashed Lines. 


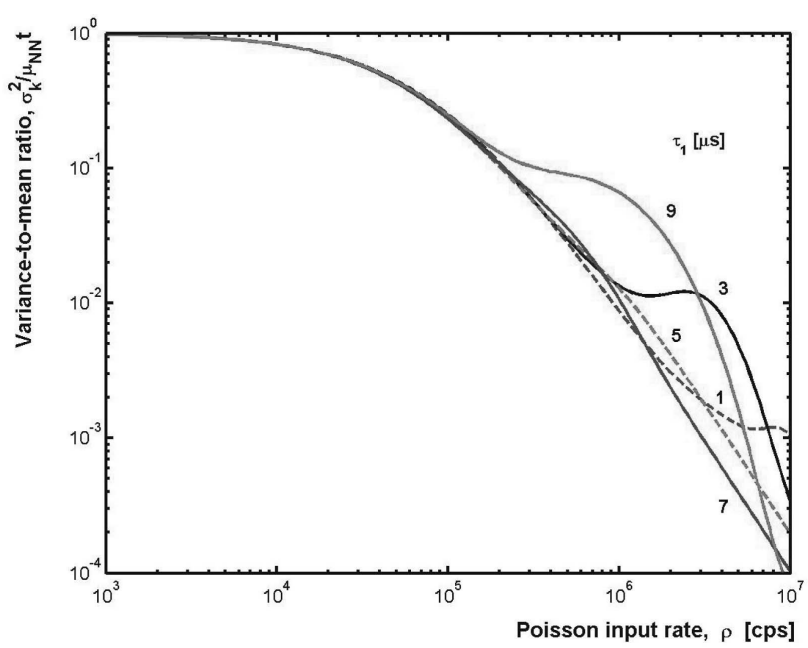

Fig. 11. (Color Online) Variance-to-mean Ratio $\sigma_{\mathrm{k}}{ }^{2} / \mu_{\mathrm{NN}} \mathrm{t}$ for the NE-NE Series DTs Under an Asymptotic Equilibrium Counting. The Poisson Input Rate $\rho$ was Varied, whereas the Second DT, $\tau_{2}=10 \mu \mathrm{s}$, and Counting Time, $t=1 \mathrm{~s}$, were Fixed for an Asymptotic Limit.

can be confirmed by applying Eqs. (12)-(13) to the counting rates at position-2 $[1,14,36]$. Similarly, the equilibrium mean counting rates $\mu_{\mathrm{ne}}$ and $\mu_{\mathrm{ee}}$ obtained in the previous works can also be verified $[14,40]$. Eqs. (12) and (13) can also be used to obtain the equilibrium counting rates of the Poisson process modified by three DTs in series. The required TID function ${ }_{2} f(\mathrm{t})$ and total density ${ }_{2} D(\mathrm{t})$ are listed in the appendices of the present study and ref. [40]. By further abbreviating the notation $\mathrm{NE}$ to $\mathrm{N}$ to avoid confusion, there are 8 possible cases - ENE, ENN, NNE, NNN, NEE, NEN, EEE, and EEN, from a combination of three DTs in series. To simplify the integrations in Eqs. (12) and (13), only the results are listed in Appendix C. A practical application of the derived counting rates remains to be examined.

\section{CONCLUSION}

Counting statistics modified by a series combination of two DTs was fully developed and discussed for the interval density, total density, expected number of counts and variance in a given time interval $(0, t)$; asymptotic expressions for the ordinary, equilibrium and shifted processes were also determined. The cases studied are a combination of the E-NE (extended-nonextended) DTs and the NE-NE (nonextended-nonextended) DTs. The other two cases of combinations NE-E and E-E DTs were dealt with in a previous study. Whenever an input pulse sequence with a constant total event density after a period of no events is modified by an NE-type dead time, the modified event TID is the shifted one of the input TID. For modification by the E-type dead time, in which the input TID has an exponential shape, the shape of the modified total density is shifted from the input total density. The equilibrium mean counting rates for the 8 cases modified by the three DTs in series - ENE, ENN, NNE, NNN, NEE, NEN, EEE, and EEN were briefly derived. A possible practical application of the derived formulae is discussed.

\section{APPENDIX}

The Appendices list the derived formulae. Several abbreviations were used to simplify the equations, most of which were taken from Müller [14,15] and from a preceding study [40]. The shifted Poisson probability $\mathrm{P}_{\mathrm{s}}\left(\mathrm{j}, \mathrm{Z}_{\mathrm{k}}\right)$ is given as: $P_{S}\left(j, z_{k}\right) \equiv\left(z_{k}^{j} / j !\right) \exp \left(-z_{k}\right)$ with the convention of $P_{s}\left(j=0, z_{k}=0\right)=1$. In addition, a set of characteristic quantities relevant to the NE-NE series DT is defined as:

$$
\overline{Q_{n}} \equiv \sum_{j=n}^{J} P_{S}\left(j-n, \hat{x}_{j}\right)=\sum_{j=n}^{J} P_{S}\left(j-n, x_{2}-j x_{1}\right),
$$

for integers $\mathrm{n} \leq \mathrm{J}$. This definition is similar to the quantity $\mathrm{Q}_{\mathrm{n}}$ defined for the case of the extended-extended (E-E) series DT [40].

$$
\begin{aligned}
& T_{k r} \equiv \rho\left[t-\tau_{2}-k \tau_{1}-r\left(\tau_{2}-\tau_{1}\right)\right], \\
& T_{k} \equiv T_{k 0}=\rho\left(t-\tau_{2}-k \tau_{1}\right), \\
& K \equiv \max \left(\left[\left[\frac{t-\tau_{2}}{\tau_{1}}\right]\right], 0\right), \\
& x_{1} \equiv \rho \tau_{1}, x_{2} \equiv \rho \tau_{2}, x \equiv x_{2}-x_{1}, \\
& g_{1} \equiv e^{-\rho \tau_{1}}=e^{-x_{1}}, \lambda_{e n} \equiv \frac{1}{1+x g_{1}}, \\
& \mu_{e n} \equiv \rho g_{1} \lambda_{e n} ; \\
& J \equiv\left[\left[\frac{\tau_{2}}{\tau_{1}}\right]\right], \quad c_{k r j} \equiv(J+1)(k-r)+j, \\
& M=M_{k r j q} \equiv c_{k r j}+q, \\
& T_{k r j} \equiv \rho\left[t-\left(c_{k r j}+r\right) \tau_{1}-r\left(\tau_{2}-\tau_{1}\right)\right], \\
& \hat{T}_{j} \equiv T_{00 j}=\rho\left(t-j \tau_{1}\right), \hat{x}_{j} \equiv \rho\left(\tau_{2}-j \tau_{1}\right)=x_{2}-j x_{1}, \\
& \tilde{K} \equiv \max \left(\left[\left[\frac{t}{\tau_{1}}\right]\right],\left[\left[\frac{t}{\tau_{2}-\tau_{1}}\right]\right]\right), \\
& \lambda_{1} \equiv \frac{1}{1+x_{1}}, \quad \lambda_{N N} \equiv\left[1+J-\sum_{n=1}^{J} \overline{Q_{n}}\right]^{-1} \\
& \mu_{N N} \equiv \rho \lambda_{1} \lambda_{N N} .
\end{aligned}
$$

\section{A. E-NE Series Dead Time}

The density functions are:

$$
\begin{aligned}
& { }_{2} f(t)=\rho g_{1} \sum_{k=0}^{K} U\left(T_{k}\right) \frac{\left(-g_{1} T_{k}\right)^{k}}{k !}={ }_{1}^{E} f\left(t-\tau_{2}+\tau_{1}\right) \\
& { }_{2} D(t)=\rho g_{1} \sum_{k=0}^{K} \sum_{r=0}^{k}\left(\begin{array}{l}
k \\
r
\end{array}\right)(-1)^{k+r} U\left(T_{k r}\right) \frac{\left(g_{1} T_{k r}\right)^{k}}{k !}
\end{aligned}
$$


and:

$$
\begin{gathered}
{ }_{2} f^{*}(s)=\frac{\rho e^{-\rho \tau_{1}} e^{-\tau_{2} s}}{s+\rho e^{-\tau_{1}(s+\rho)}} \\
{ }_{2} D^{*}(s)=\frac{\rho e^{-\rho \tau_{1}} e^{-\tau_{2} s}}{s+\rho e^{-\rho \tau_{1}}\left(e^{-\tau_{1} s}-e^{-\tau_{2} s}\right)}
\end{gathered}
$$

where the binomial coefficient is $\left(\begin{array}{l}k \\ r\end{array}\right)=k ! / r !(k-r)$ ! with the convention of $\left(\begin{array}{l}0 \\ 0\end{array}\right) \equiv 1$.

\section{A.1. Ordinary Process}

Counting statistics (exact):

$$
\begin{aligned}
& E(k, t) \equiv \hat{k}(t)=\sum_{k=0}^{K} \sum_{r=0}^{k}\left(\begin{array}{l}
k \\
r
\end{array}\right)(-1)^{k+r} U\left(T_{k r}\right) \frac{\left(g_{1} T_{k+}\right)^{k+1}}{(k+1) !} \\
& V(k, t) \equiv \sigma_{k}^{2}(t)=2 \int_{0}^{t} D(\lambda) \hat{k}(t-\lambda) d \lambda+\hat{k}(t)-[\hat{k}(t)]^{2}
\end{aligned}
$$

where $\hat{k}(t)$ is given in Eq. (A.8). The numerical convolution of ${ }_{2} D(\mathrm{t})$ and $\hat{k}(t)$ is easier to obtain because the resulting expression for the variance of counts $\sigma_{k}^{2}(t)$ in Eq. (A.9) is long and complicated.

Asymptotic expressions:

$$
\begin{gathered}
\hat{k}(t) \cong \mu_{e n} t-\lambda_{e n}^{2} g_{1} x_{2}-\frac{1}{2}\left(1-\lambda_{e n}\right)^{2} \\
\sigma_{k}^{2}(t) \cong \mu_{e n} \lambda_{e n}^{2}\left(1-2 g_{1} x_{1}\right) t \\
+\frac{1}{12} \lambda_{e n}^{4}\left\{g_{1}^{4}\left(x_{2}-x_{1}\right)^{4}+4 g_{1}^{3}\left(x_{2}-x_{1}\right)^{3}\right. \\
\left.+6 g_{1}^{2}\left(x_{2}^{2}+6 x_{2} x_{1}-x_{1}^{2}\right)-12 g_{1} x_{2}\right\} .
\end{gathered}
$$

\section{A.2. Equilibrium Process}

Counting statistics (exact):

$$
\begin{gathered}
E(k, t) \equiv \hat{k}(t)=\mu_{e n} t \\
V(k, t) \equiv \sigma_{k}^{2}(t)=2 \lambda_{e n} \sum_{k=0}^{K} \sum_{r=0}^{k}\left(\begin{array}{l}
k \\
r
\end{array}\right)(-1)^{k+r} U\left(T_{k r}\right) \frac{\left(g_{1} T_{k r}\right)^{k+2}}{(k+2) !} \\
+\mu_{e n} t-\left(\mu_{e n} t\right)^{2}
\end{gathered}
$$

Asymptotic expression:

$$
\begin{aligned}
\sigma_{k}^{2}(t) \cong & \mu_{e n} \lambda_{e n}^{2}\left(1-2 g_{1} x_{1}\right) t \\
& +\frac{1}{6} \lambda_{e n}^{4} g_{1}^{2}\left\{g_{1}^{2}\left(x_{2}-x_{1}\right)^{4}\right. \\
& \left.+2 g_{1}\left(x_{2}-x_{1}\right)\left(2 x_{2}^{2}-4 x_{2} x_{1}-x_{1}^{2}\right)+6 x_{2}^{2}\right\} .
\end{aligned}
$$

\section{A.3. Shifted Process}

Counting statistics (exact):

$$
\begin{aligned}
E(k, t) & \equiv \hat{k}(t) \\
& =U(t)\left[1-e^{-\rho t}\right] \\
& +\sum_{k=0}^{K} \sum_{r=0}^{k}\left(\begin{array}{l}
k \\
r
\end{array}\right)(-1)^{r} g_{1}^{k+1} U\left(T_{k r}\right) e^{-T_{k r}}\left\{1-\sum_{j=0}^{k+1} P_{S}\left(j,-T_{k r}\right)\right\}
\end{aligned}
$$

$$
V(k, t) \equiv \sigma_{k}^{2}(t)=2 \int_{0}^{t} D(\lambda) \hat{k}(t-\lambda) d \lambda+\hat{k}(t)-[\hat{k}(t)]^{2}
$$

where $\hat{k}(t)$ is given in Eq. (A.15). We suggest a numerical convolution of ${ }_{2} D(\mathrm{t})$ and $\hat{k}(t)$.

Asymptotic expressions:

$$
\begin{aligned}
\hat{k}(t) \cong \mu_{e n} t & -\lambda_{e n} g_{1} \\
+ & \frac{1}{2} \lambda_{e n}^{2}\left\{g_{1}^{2}\left(x_{2}-x_{1}\right)^{2}+2 g_{1}\left(x_{2}-2 x_{1}\right)+2\right\} \\
\sigma_{k}^{2}(t) \cong \mu_{e n} & \lambda_{e n}^{2}\left(1-2 g_{1} x_{1}\right) t \\
+\lambda_{e n}^{4} & \left\{g_{1}^{4}\left[\frac{1}{12}\left(x_{2}-x_{1}\right)^{4}+\left(x_{2}-x_{1}\right)^{2}\right]\right. \\
& +g_{1}^{3}\left[\frac{1}{3}\left(x_{2}-x_{1}\right)^{3}+2\left(x_{2}-x_{1}\right)\left(1+x_{1}-x_{1}^{2}\right)\right] \quad \text { A. } \\
+ & g_{1}^{2}\left[\frac{1}{2}\left(x_{2}-x_{1}\right)^{2}-\left(x_{2}-x_{1}\right)\left(1-4 x_{1}\right)\right. \\
& \left.\left.+x_{1}\left(2+3 x_{1}\right)+1\right]-g_{1}\left(1+x_{2}\right)\right\} .
\end{aligned}
$$

\section{B. NE-NE Series Dead Times}

The density function is obtained from the integration of Eq. (3):

$$
\begin{aligned}
& { }_{2} f(t)=\left\{U\left(T_{0}\right)-U\left(T_{1}\right)\right\}{ }_{1}^{N E} D(t) \\
& +U\left(T_{1}\right) \rho e^{-\rho\left(t-\tau_{2}-\tau_{1}\right)} \bar{Q}_{0}
\end{aligned}
$$

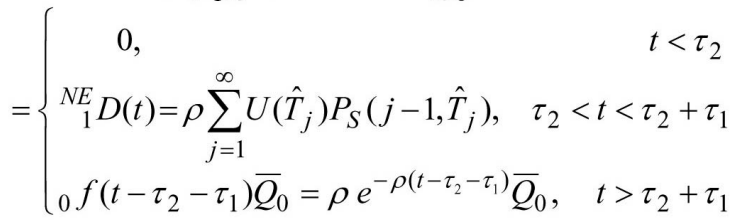

$$
\begin{aligned}
& { }_{2} D(t)=\rho \sum_{k=1}^{\tilde{K}} \sum_{r=0}^{k} \sum_{j=0}^{r} \sum_{q=r}^{r J}\left(\begin{array}{l}
k \\
r
\end{array}\right)\left(\begin{array}{l}
r \\
j
\end{array}\right)(-1)^{j} \\
& B_{r q} U\left(T_{k r j}\right) P_{S}\left(c_{k r j}+q-1, T_{k r j}\right) .
\end{aligned}
$$

The transformed density functions can be expressed as:

$$
\begin{aligned}
& { }_{2} f^{*}(s)=e^{-\tau_{2} s}\left[1-\frac{\rho e^{-\tau_{1} s}}{\rho+s}\right]\left\{\sum_{n=1}^{J}\left(\frac{\rho}{\rho+s}\right)^{n} \bar{Q}_{n}\right\}+\left[\frac{\rho e^{-\tau_{1} s}}{\rho+s}\right]^{J+1} \\
& { }_{2} D^{*}(s)=\sum_{k=1}^{\infty} \sum_{r=0}^{k} \sum_{j=0}^{r} \sum_{q=r}^{r J}\left(\begin{array}{l}
k \\
r
\end{array}\right)\left(\begin{array}{l}
r \\
j
\end{array}\right)(-1)^{j} B_{r q}\left(\frac{\rho}{\rho+s}\right)^{c_{k j}+q} e^{-\left(c_{k j} \tau_{1}+r \tau_{2}\right) s}
\end{aligned}
$$

where the $\mathrm{Brq}$ 's are given as:

$$
B_{r q}=\sum_{\left\{r_{n}\right\}} \frac{r !}{r_{1} ! \cdots r_{J} !} \prod_{n=1}^{J} \bar{Q}_{n}^{r_{n}}
$$

with the sum over all possible sets $\left\{\mathrm{r}_{\mathrm{n}}\right\}$ under the conditions: $q=\sum_{n} n r_{n}$ and $r=\sum_{n} r_{n}$. It is easily seen that $\mathrm{B}_{00}=1$. Instead of finding all possible sets, $\left\{\mathrm{r}_{\mathrm{n}}\right\}$, and calculating the multinomial coefficients in Eq. (B.5), the following recurrence relation makes it easier to generate $B_{r q}$ successively for increasing $r$ starting from $r=1$ and using $\mathrm{B}_{00}=1 . \mathrm{B}_{\mathrm{rq}}$ can be expressed as [40]:

$$
B_{r q}=\sum_{n=n_{1}}^{n_{2}} B_{r-1, q-n} \bar{Q}_{n},
$$


where $\mathrm{n}_{1}=\max [1, \mathrm{q}-(\mathrm{r}-1) \mathrm{J}], \mathrm{n}_{2}=\min [\mathrm{q}-\mathrm{r}+1, \mathrm{~J}]$ and $\mathrm{q}$ running from $\mathrm{r}$ to $\mathrm{rJ}$ for $\mathrm{r}=1, \ldots, \mathrm{k}$.

The asymptotic variance of the counts is obtained in terms of the r-th moments of the time interval $m_{r}(t)$ and by the Elmore theorem $[44,47]$. The time independent parts in the final expression for the variances are too long to list, and are listed separately in terms of the $1^{\text {st }}, 2^{\text {nd }}$ and the $3^{\text {rd }}$ moments, $\mathrm{m}_{1}, \mathrm{~m}_{2}, \mathrm{~m}_{3}$, of the TID function, $2 f(\mathrm{t})$, given in Eq. (B.1). The moments are expressed as follows:

$$
\begin{gathered}
m_{1}=\left(\rho \lambda_{1} \lambda_{N N}\right)^{-1}=1 / \mu_{N N} \\
m_{2}=\frac{1}{\left(\rho \lambda_{1}\right)^{2}}\left\{\begin{array}{l}
(J+1)^{2}+(J+1) \lambda_{1}^{2} \\
\left.-\left(\lambda_{1}^{2}+2 \lambda_{1} x_{2}+1\right)\left(\sum_{n=1}^{J} \bar{Q}_{n}\right)-2 \lambda_{1}\left(\sum_{n=1}^{J} n \bar{Q}_{n}\right)\right\}
\end{array}\right. \\
m_{3}=\frac{1}{\rho^{3}}\left\{(J+1)^{3}\left(x_{1}+1\right)^{3}+3(J+1)^{2}\left(x_{1}+1\right)+2(J+1)\right\} \\
-\frac{1}{\rho^{3}}\left\{\left(x_{1}+1\right)^{3}+3 x_{2}\left(x_{1}+1\right)^{2}\right. \\
\left.+3\left(x_{2}^{2}+1\right)\left(x_{1}+1\right)+3 x_{2}+2\right\}\left(\sum_{n=1}^{J} \bar{Q}_{n}\right) \\
-\frac{3}{\rho^{3}}\left\{\left(x_{1}+1\right)^{2}+\left(2 x_{2}+1\right)\left(x_{1}+1\right)\right. \\
+1\}\left(\sum_{n=1}^{J} n \overline{Q_{n}}\right)-\frac{3}{\rho^{3}}\left(x_{1}+1\right)\left(\sum_{n=1}^{J} n^{2} \bar{Q}_{n}\right) .
\end{gathered}
$$

\section{B.1. Ordinary Process}

Counting statistics (exact):

$$
\begin{aligned}
E(k, t) \equiv \hat{k}(t) & \text { (B.10) } \\
& =\sum_{k=1}^{\widetilde{K}} \sum_{r=0}^{k} \sum_{j=0}^{r} \sum_{q=r}^{r J}\left(\begin{array}{l}
k \\
r
\end{array}\right)\left(\begin{array}{l}
r \\
j
\end{array}\right)(-1)^{j} B_{r q} U\left(T_{k r j}\right)\left\{1-\sum_{n=0}^{M-1} P_{S}\left(n, T_{k r j}\right)\right\}
\end{aligned}
$$$$
V(k, t) \equiv \sigma_{k}^{2}(t)=2 \int_{0}^{t} D(\lambda) \hat{k}(t-\lambda) d \lambda+\hat{k}(t)-[\hat{k}(t)]^{2}
$$

where $\hat{k}(t)$ is given in Eq. (B.10). Since the resulting expression for the variance of counts $\sigma_{\mathrm{k}}{ }^{2}(\mathrm{t})$ in Eq. (B.11) contains multiple sums, a numerical convolution of ${ }_{2} D(\mathrm{t})$ and $\hat{k}(t)$ is suggested.

Asymptotic expressions:

$$
\begin{gathered}
\hat{k}(t) \cong \mu_{N N} t-1+\frac{1}{2} \lambda_{N N}\left\{\lambda_{1}^{2}-\lambda_{N N} \sum_{k=1}^{J}\left[1+2 \lambda_{1}\left(x_{2}+k\right)\right] \bar{Q}_{k}\right. \\
\left.+(J+1)^{2} \lambda_{N N}\right\} \\
\sigma_{k}^{2}(t) \cong \mu_{N N} t\left\{\lambda_{1}^{2} \lambda_{N N}-\lambda_{N N}^{2} \sum_{k=1}^{J}\left[1+2 \lambda_{1}\left(x_{2}+k\right)\right] \overline{Q_{k}}\right. \\
\left.+(J+1)^{2} \lambda_{N N}^{2}-1\right\} \\
+\frac{1}{m_{1}^{2}}\left(-\frac{m_{2}}{2}-\frac{2 m_{3}}{3 m_{1}}+\frac{5 m_{2}^{2}}{4 m_{1}^{2}}\right) .
\end{gathered}
$$

\section{B.2. Equilibrium Process}

Counting statistics (exact):

$$
\begin{gathered}
E(k, t) \equiv \hat{k}(t)=\mu_{N N} t \\
V(k, t) \equiv \sigma_{k}^{2}(t)=2 \lambda_{1} \lambda_{N N} \sum_{k=1}^{\tilde{K}} \sum_{r=0}^{k} \sum_{j=0}^{r}\left(\begin{array}{l}
k \\
r
\end{array}\right)\left(\begin{array}{r}
r \\
j
\end{array}\right)(-1)^{j} U\left(T_{k r j}\right) \times \\
\left\{T_{k r j}\left(\sum_{n=1}^{J} \bar{Q}_{n}\right)^{r}-\sum_{q=r}^{r J} B_{r q} \sum_{m=0}^{M-1}\left[1-\sum_{n=0}^{m} P_{S}\left(n, T_{k r j}\right)\right]\right\} \\
+\left(\mu_{N N} t\right)-\left(\mu_{N N} t\right)^{2}
\end{gathered}
$$

Asymptotic expression:

$$
\begin{array}{r}
\sigma_{k}^{2}(t) \cong \mu_{N N} t\left\{\lambda_{1}^{2} \lambda_{N N}-\lambda_{N N}^{2} \sum_{k=1}^{J}\left[1+2 \lambda_{1}\left(x_{2}+k\right)\right] \overline{Q_{k}}\right. \\
\left.+(J+1)^{2} \lambda_{N N}^{2}-1\right\}+\frac{1}{m_{1}^{3}}\left(\frac{m_{2}^{2}}{2 m_{1}}-\frac{m_{3}}{3}\right) .
\end{array}
$$

\section{B.3. Shifted Process}

Counting statistics (exact):

$$
\begin{aligned}
& E(k, t) \equiv \hat{k}(t) \\
&=\sum_{k=0}^{\tilde{K}} \sum_{r=0}^{k} \sum_{j=0}^{r} \sum_{q=r}^{r J}\left(\begin{array}{l}
k \\
r
\end{array}\right)\left(\begin{array}{l}
r \\
j
\end{array}\right)(-1)^{j} B_{r q} U\left(T_{k r j}\right)\left\{1-\sum_{n=0}^{M} P_{S}\left(n, T_{k r j}\right)\right\} \\
& V(k, t) \equiv \sigma_{k}^{2}(t)=2 \int_{0}{ }_{2} D(\lambda) \hat{k}(t-\lambda) d \lambda+\hat{k}(t)-[\hat{k}(t)]^{2}
\end{aligned}
$$

where $\hat{k}(t)$ is given in Eq. (B.17). We suggest a numerical convolution of ${ }_{2} D(\mathrm{t})$ and $\hat{k}(t)$.

Asymptotic expressions:

$$
\begin{gathered}
\hat{k}(t) \cong \mu_{N N} t+\frac{1}{2} \lambda_{N N}\left\{\begin{array}{r}
\lambda_{1}^{2}-2 \lambda_{1}-\lambda_{N N} \sum_{k=1}^{J}\left[1+2 \lambda_{1}\left(x_{2}+k\right)\right] \bar{Q}_{k}(\mathrm{~B} .19) \\
\left.+(J+1)^{2} \lambda_{N N}\right\}
\end{array}\right. \\
\sigma_{k}^{2}(t) \cong \mu_{N N} t\left\{\lambda_{1}^{2} \lambda_{N N}-\lambda_{N N}^{2} \sum_{k=1}^{J}\left[1+2 \lambda_{1}\left(x_{2}+k\right)\right] \bar{Q}_{k}\right. \\
\left.+(J+1)^{2} \lambda_{N N}^{2}-1\right\} \\
+\frac{1}{m_{1}}\left[\frac{1}{\rho}-\frac{1}{m_{1}}\left(\frac{m_{2}}{2}-\frac{1}{\rho^{2}}\right)\right. \\
\left.-\frac{1}{m_{1}^{2}}\left(\frac{m_{2}}{\rho}+\frac{2 m_{3}}{3}\right)+\frac{5 m_{2}^{2}}{4 m_{1}^{3}}\right] .
\end{gathered}
$$

\section{Mean Count Rates Modified by Three Dead Times in Series}

The definitions of the quantities $\bar{Q}_{n}$ in Eq. (A.1) and $Q_{n}$ in Eq. (A.3) of the previous study [40] can be generalized by adding parentheses to accommodate the general arguments for $u$ and $v$ as follows:

$$
\begin{aligned}
& \overline{Q_{n}}(J ; u, v) \equiv \sum_{j=n}^{J} P_{S}(j-n, u-j v), \\
& Q_{n}(J ; u, v) \equiv \sum_{j=n}^{J} P_{S}(j-n,-u+j v) .
\end{aligned}
$$

Whenever there are no arguments in $\bar{Q}_{n}$ or $Q_{n}$, the arguments 
$u=x_{2}$ and $v=x_{1}$ are assumed in order to maintain consistency with the definitions in the present and previous studies [40]. In addition to the abbreviations defined in Eqs. (A.1)-(A.3), several new abbreviations are added, as follows:

$$
\begin{aligned}
& J_{3} \equiv\left[\left[\frac{\tau_{3}}{\tau_{1}}\right]\right], K_{3} \equiv\left[\left[\frac{\tau_{3}-\tau_{2}}{\tau_{1}}\right]\right], \\
& \tilde{K}_{3} \equiv \max \left(\left[\left[\frac{\tau_{3}}{\tau_{1}}\right]\right],\left[\left[\frac{\tau_{3}}{\tau_{2}-\tau_{1}}\right]\right]\right), \\
& x_{3} \equiv \rho \tau_{3}, g_{3} \equiv e^{-\rho \tau_{3}}=e^{-x_{3}}, g \equiv e^{-\rho\left(\tau_{2}-\tau_{1}\right)}=g_{2} / g_{1}, \\
& \hat{\tau}_{k} \equiv \rho\left(\tau_{3}-k \tau_{1}\right)=x_{3}-k x_{1}, \\
& \hat{\tau}_{k r} \equiv \rho\left[\tau_{3}-\tau_{2}-k \tau_{1}-r\left(\tau_{2}-\tau_{1}\right)\right]=x_{3}-x_{2}-k x_{1}-r\left(x_{2}-x_{1}\right), \\
& \hat{\tau}_{k r j} \equiv \rho\left[\tau_{3}-\left(c_{k r j}+r\right) \tau_{1}-r\left(\tau_{2}-\tau_{1}\right)\right]=x_{3}-r x_{2}-c_{k r j} x_{1} .
\end{aligned}
$$

\section{C.1. Extended-Nonextended-Extended (ENE) Series DTs}

$$
\mu_{E N E}=\mu_{e n}\left(g_{1} g_{3} / g_{2}\right) Q_{0}\left(K_{3}+1 ; x_{3}-x_{2}+x_{1}, x_{1}\right)
$$

\section{C.2. Extended-Nonextended-Nonextended (ENN)} Series DTs

$\mu_{E N N}=\mu_{e n}\left\{1+\sum_{k=0}^{K_{3}} \sum_{r=0}^{k} U\left(\hat{\tau}_{k r}\right)\left(\begin{array}{l}k \\ r\end{array}\right)(-1)^{k+r} \frac{\left(g_{1} \hat{\tau}_{k r}\right)^{k+1}}{(k+1) !}\right\}^{-1}$

\section{C.3. Nonextended-Nonextended-Extended (NNE) Series DTs}

$$
\begin{gathered}
\mu_{N N E}=\mu_{N N}\left[1-\left(J_{3}-J\right)+\sum_{n=1}^{J_{3}} \bar{Q}_{n}\left(J_{3} ; x_{3}, x_{1}\right)\right. \\
\left.-\sum_{n=1}^{J} \bar{Q}_{n}\left(J ; x_{2}, x_{1}\right)\right], \tau_{2}<\tau_{3}<\tau_{2}+\tau_{1} \\
\mu_{N N E}=\mu_{N N} \frac{g_{3}}{g_{1} g_{2}} \bar{Q}_{0}, \quad \tau_{3} \geq \tau_{2}+\tau_{1}
\end{gathered}
$$

\section{C.4. Nonextended-Nonextended-Nonextended} (NNN) Series DTs

$$
\begin{array}{r}
\mu_{N N N}=\mu_{N N} \\
\left\{1+\sum_{k=1}^{\tilde{K}_{3}} \sum_{r=0}^{k} \sum_{j=0}^{r} \sum_{q=r}^{r J}\left(\begin{array}{l}
k \\
r
\end{array}\right)\left(\begin{array}{l}
r \\
j
\end{array}\right)(-1)^{j} B_{r q} U\left(\hat{\tau}_{k r j}\right)\right. \\
\left.\left[1-\sum_{n=0}^{M-1} P_{S}\left(n, \hat{\tau}_{k r j}\right)\right]\right\}^{-1}
\end{array}
$$

C.5. Nonextended-Extended-Extended (NEE) Series DTs

$\mu_{N E E}=\mu_{n e}\left[1+\sum_{k=0}^{K_{3}} \sum_{r=0}^{k}\left(\begin{array}{l}k \\ r\end{array}\right)(-g)^{r+1} U\left(\hat{\tau}_{k r}\right)\left\{1-\sum_{n=0}^{k} P_{S}\left(n, \hat{\tau}_{k r}\right)\right\}\right]$

\section{C.6. Nonextended-Extended-Nonextended (NEN) Series DTs}

$\mu_{N E N}=\mu_{n e}\left\{1+g\left[\left(K_{3}+1\right)-\sum_{n=0}^{K_{3}} \bar{Q}_{n}\left(K_{3} ; x_{3}-x_{2}, x_{1}\right)\right]\right\}^{-1}$

\section{C.7. Extended-Extended-Extended (EEE) Series DTs}

$$
\begin{aligned}
\mu_{E E E}=\mu_{e e}\left[1-\sum_{k=1}^{\tilde{K}_{3}} \sum_{r=0}^{k} \sum_{j=0}^{r} \sum_{q=r}^{r J}\left(\begin{array}{l}
k \\
r
\end{array}\right)\left(\begin{array}{l}
r \\
j
\end{array}\right)(-1)^{j+1}\right. \\
\left.A_{r q} U\left(\hat{\tau}_{k r j}\right) e^{-\left(c_{k j} x_{1}+x_{2}\right)} \frac{\left(-\hat{\tau}_{k r j}\right)^{c_{k j}+q}}{\left(c_{k r j}+q\right) !}\right]
\end{aligned}
$$

\section{C.8. Extended-Extended-Nonextended (EEN) Series} DTs

$$
\begin{array}{r}
\mu_{E E N}=\mu_{e e}\left\{1+g_{2} Q_{0}\left(J ; x_{2}, x_{1}\right)-g_{3} Q_{0}\left(J ; x_{3}, x_{1}\right)\right. \\
\left.-U\left[\tau_{3}-(J+1) \tau_{1}\right] g_{3} P_{S}\left(J+1,-\hat{\tau}_{J+1}\right)\right\}^{-1}, \\
\tau_{2}<\tau_{3}<\tau_{2}+\tau_{1}
\end{array}
$$

\section{ACKNOWLEDGEMENTS}

The author wishes to acknowledge Dr. S. Pommé for his kind provision of the Monte Carlo code. Dr. C. Berglöf also kindly shared his experimental data, which is related with the E-NE series DT model. Thanks are also due to Mr. B. G. Park for preparing the figures.

\section{REFERENCES}

[ 1 ] R. Jost, "Bemerkungen zur mathematischen Theorie der Zähler," Helv. Phys. Acta. 20, pp. 173-182 (1947).

[2] W. Feller, "On Probability Problems in the Theory of Counters," Courant Anniversary Volume, pp. 105-115, Interscience, New York (1948).

[ 3 ] NCRP Report No. 58, "A Handbook of Radioactivity Measurements Procedures," $2^{\text {nd }}$ ed., p. 60, National Council on Radiation Protection and Measurements, Bethesda, MD, USA (1985).

[ 4 ] G. F. Knoll, Radiation Detection and Measurement, $3^{\text {rd }}$ ed., pp. 119-127, John Wiley \& Sons, New York (1989).

[5] ICRU Report No. 52, "Particle Counting in Radioactivity Measurements," p. 80, International Commission on Radiation Units and Measurements, Bethesda, MD, USA (1994).

[6] A. E. Ruark and F. E. Brammer, "The Efficiency of Counters and Counter Circuits," Phys. Rev. 52, pp. 322-324 (1937).

[7] L. Kosten, "On the frequency distribution of the number of discharges counted by a Geiger-Müller counter in a constant interval," Physica X, no. 9, pp. 749-756 (1943).

[8] L. L. Campbell, "Standard Deviation of Dead Time Correction in Counters," Can. J. Phys. 34, pp. 929-937 (1956).

[9] I. De Lotto, P. F. Manfredi, P. Principi, "Counting statistics and dead-time losses, Part 1," Energia Nucleare 11, pp. 557-564 (1964).

[10] J. W. Müller, "On the interval-distribution for recurrent events with a non-extended dead time," Report BIPM105, Bureau International des Poids et Mesures, Sèvres, France (1967).

[11] A. F. Para, M. M. Bettoni, "Counting statistics of nuclear detectors," Nucl. Instr. and Meth. 70, pp. 52-56 (1969).

[12] J. W. Müller, "Counting statistics of a Poisson process 
with dead time," Report BIPM-111, Bureau International des Poids et Mesures, Sèvres, France (1970).

[13] J. W. Müller, "Interval densities for extended dead times," Report BIPM-112, Bureau International des Poids et Mesures, Sèvres, France (1971).

[14] J. W. Müller, "Dead-time problems," Nucl. Instr. and Meth. 112, pp. 47-57 (1973).

[15] J. W. Müller, "Some formulae for a dead-time-distorted Poisson process: To André Allisy on the completion of his first half century," Nucl. Instr. and Meth. 117, pp. 401-404 (1974).

[16] J. Libert, "Statistique de comptage : À propos d'une expérience récente," Nucl. Instr. and Meth. 126, pp. 589590 (1975).

[17] D. R. Cox, Renewal Theory, Methuen, London (1962).

[18] S. Pommé, B. Denecke, J.-P. Alzetta, "Influence of pileup rejection on nuclear counting, viewed from the time-domain perspective," Nucl. Instr. and Meth. in Phys. Res. A 426, pp. 564-582 (1999).

[19] D. F. Cowell, M. M. Sandomire, M. S. Eichen, "Automatic Compensation of Dead Time in Pulse Analysis Equipment," Anal. Chem. 32, pp. 1086-1090 (1960).

[20] M. O. Deighton, "Statistical errors arising from use of a gated pulse train for total live time measurement during pulse amplitude analysis," Nucl. Instr. and Meth. 14, pp. 48-52 (1961).

[21] O. U. Anders, "Experiences with the Ge(Li) detector for high-resolution gamma ray spectrometry and a practical approach to the pulse pileup problem," Nucl. Instr. and Meth. 68, pp. 205-208 (1969).

[22] M. Wiernik, "Normal and random pulse generators for the correction of dead-time losses in nuclear spectrometry," Nucl. Instr. and Meth. 96, pp. 325-329 (1971).

[23] J. Harms, "Automatic dead-time correction for multichannel pulse-height analyzers at a variable counting rates," Nucl. Instr. and Meth. 53, pp. 192-196 (1967).

[24] G. P. Westphal, "On the performance of loss-free counting - A method for real-time compensation of dead-time and pile-up losses in nuclear pulse spectroscopy," Nucl. Instr. and Meth. 163, pp. 189-196 (1979).

[25] E. Schönfeld, H. Janssen, "Precise measurement of dead time," Nucl. Instr. and Meth. in Phys. Res. A 339, pp. 137143 (1994).

[26] J. Bouchard, "MTR2: A discriminator and dead-time module used in counting systems," Appl. Radiat. Isotopes 52, pp. 441-446 (2000).

[27] C. Michotte, M. Nonis, "Experimental comparison of different dead-time correction techniques in single-channel counting experiments," Nucl. Instr. and Meth. in Phys. Res. A 608, pp. 163-168 (2009).

[28] K. R. D. Mylon, G. W. McBeth, "Radioactive decay viewed through an extending dead time," Nucl. Instr. and Meth. in Phys. Res. 217, pp. 459-464 (1983).

[29] M. M. R. Williams, Random Processes in Nuclear Reactors, pp. 26-49, Pergamon, Oxford (1974).

[30] T. Hazama, "Practical correction of dead time effect in variance-to-mean ratio measurement," Annals of Nuclear
Energy 30, pp. 615-631 (2003).

[31] C. Berglöf, M. Fernández-Ordóñez, D. Villamarín, V. Bécares, E.M. González-Romero, V. Bournos, J.-L. MuñozCobo, "Auto-correlation and variance-to-mean measurements in a subcritical core obeying multiple alpha-modes," Annals of Nuclear Energy 38, pp. 194-202 (2011).

[32] C. E. Cohn, "The effect of deadtime on counting errors," Nucl. Instr. and Meth. 41, pp. 338-340 (1966).

[33] S. Pommé, "Cascades of pile-up and dead time," Appl. Radiat. Isotopes 66, pp. 941-947 (2008).

[34] J. W. Müller, ed., "Bibliography on dead-time effects," Report BIPM-81/11, Bureau International des Poids et Mesures, Sèvres, France (1981).

[35] E. Funck, "Dead time effects from linear amplifiers and discriminators in single detector systems," Nucl. Instr. and Meth. in Phys. Res. A 245, pp. 519-524 (1986).

[36] I. De Lotto, P. F. Manfredi, P. Principi, "Counting statistics and dead-time losses, Part 2," Energia Nucleare 11, pp. 599-611 (1964).

[37] I. De Lotto, P. F. Manfredi, "Counting-losses introduced by the cascade connection of two paralyzable-counters," Energia Nucleare 12, pp. 102-103 (1965).

[38] J. W. Müller, "On the influence of two consecutive dead times," Report BIPM-106, Bureau International des Poids et Mesures, Sèvres, France (1968).

[39] J. W. Müller, "On the effect of two extended dead times in series," Report BIPM-72/9, Bureau International des Poids et Mesures, Sèvres, France (1972).

[40] H. D. Choi, "Counting statistics distorted by two dead times in series which end with an extended type dead time," Nucl. Instr. and Meth. in Phys. Res. A 599, pp. 251259 (2009).

[41] J. H. Lee, I. J. Kim, H. D. Choi, "On the dead time problem of a GM counter," Appl. Radiat. Isotopes 67, pp. 1094-1098 (2009).

[42] M. Abramowitz, I. A. Stegun, Handbook of Mathematical Functions, Chap. 29, Dover Publications, Inc., New York (1970).

[43] J. W. Müller, "Une nouvelle manière de calculer les pertes de comptage dues à un temps mort non cumulatif," Report BIPM-73/4, Bureau International des Poids et Mesures, Sèvres, France (1973).

[44] W. C. Elmore, "Statistics of Counting," Nucleonics 6, n. 1, pp. 26-34 (1950).

[45] J. W. Müller, "Sur l'arrangement en sèrie de deux temps morts de types diffèrents," Report BIPM-73/9, Bureau International des Poids et Mesures, Sèvres, France (1973).

[46] J. Libert, "Statistique de comptage du compteur libre," Nucl. Instr. and Meth. 130, pp. 615-616 (1975).

[47] J. W. Müller, "Asymptotic results for a modified renewal process and their application to counting distributions," Report BIPM-77/1, Bureau International des Poids et Mesures, Sèvres, France (1977).

[48] C. Berglöf, "On Measurement and Monitoring of Reactivity in Subcritical Reactor Systems," Doctoral Thesis in Physics, KTH, Stockholm, Sweden (2010). Available at http://urn.kb.se/ resolve?urn=urn:nbn:se:kth:diva-12483. 